\title{
DUALITY, CORRESPONDENCES AND THE LEFSCHETZ MAP IN EQUIVARIANT KK-THEORY: A SURVEY
}

\author{
HEATH EMERSON
}

\begin{abstract}
We survey work by the author and Ralf Meyer on equivariant KKtheory. Duality plays a key role in our approach. We explain duality, and how duality naturally gives rise to an invariant called the Lefschetz map. With the objective of computing the Lefschetz map in explicit terms, we construct a topological model of equivariant KK-theory by way of a theory of correspondences, building on ideas of Paul Baum, Alain Connes and Georges Skandalis in the 1980's. We explain when the topological and analytic models agree, and give an example of equivariant correspondences related to the Borel-Bott-Weil theorem. Finally, we explain the relationship between the Lefschetz map and a global homological invariant using traces on appropriate K-theory groups.
\end{abstract}

The papers [13, 11, 12, 16] present a study of the equivariant Kasparov groups $\mathrm{KK}^{\mathcal{G}}\left(\mathcal{C}_{0}(X), \mathcal{C}_{0}(Y)\right)$ where $\mathcal{G}$ is a locally compact Hausdorff groupoid with Haar system and $X$ and $Y$ are $\mathcal{G}$-spaces, usually with $X$ a proper $\mathcal{G}$-space. This program builds on work of Kasparov, Connes and Skandalis done mainly in the 1980's. At that point, the main interest was the index theorem of Atiyah and Singer and its generalisations, and later, the Dirac dual-Dirac method and the Novikov conjecture. For our part, we are motivated by the idea of developing Euler characteristics and Lefschetz formulas in equivariant KK-theory. Via the Baum-Connes isomorphism - when it applies - this contributes to noncommutative topology and index theory. Our program started in [13] where we found the Lefschetz map in connection with a K-theory problem. We will give the definition of the Lefschetz map in the first section, but for now record that it has the form

$$
\text { Lef: } \mathrm{KK}_{*}^{\mathcal{G} \ltimes X}\left(\mathcal{C}_{0}\left(X \times{ }_{Z} X\right), \mathcal{C}_{0}(X)\right) \rightarrow \mathrm{KK}_{*}^{\mathcal{G}}\left(\mathcal{C}_{0}(X), \mathcal{C}_{0}(Z)\right),
$$

where we always denote by $Z$ the base space of the groupoid. This map is defined under certain somewhat technical circumstances, but, again, these normally involve proper $\mathcal{G}$-spaces $X$. The domain of the Lefschetz map is very closely related to the simpler-looking group $\mathrm{KK}_{*}^{\mathcal{G}}\left(\mathcal{C}_{0}(X), \mathcal{C}_{0}(X)\right)$ : the latter group maps in a natural way to the domain in (0.1) and this map is an isomorphism when the anchor map $X \rightarrow Z$ is a proper map. This means that the Lefschetz map can be used to assign an invariant, which is an equivariant K-homology class, to an equivariant Kasparov self-morphism of $X$. We call this class the Lefschetz invariant of the map. It bears consideration even when $\mathcal{G}$ is the trivial groupoid, and the reader can do worse than to consider this case to begin with, although by doing so one misses the applications to noncommutative topology.

The definition of (0.1) uses the notion of an abstract dual for $X$, which is an important concept playing a central role in our approach to equivariant KK-theory. Abstract duals for a given $\mathcal{G}$-space $X$ are not unique but the Lefschetz map does not depend on the choice of a dual, only on the existence of one. Abstract duals do not always exist either: a Cantor set $X$ doesn't have an abstract dual even if $\mathcal{G}$

2000 Mathematics Subject Classification. 19K35, 46L80.

Heath Emerson was supported by a National Science and Engineering Council of Canada (NSERC) Discovery grant. 
is trivial. But if $X$ is a smooth $\mathcal{G}$-manifold, with $\mathcal{G}$ acting smoothly and properly, then $X$ has an abstract dual, and if $\mathcal{G}$ is a group, then any $\mathcal{G}$-simplicial complex has an abstract dual due to 13 .

The Lefschetz map is functorial for $\mathcal{G}$-maps $X \rightarrow X^{\prime}$ in a way made explicit in $\$ 1.4$ (see Theorem 1.21). In brief, it is a homotopy invariant of the $\mathcal{G}$-space $X$. In particular, since Lef doesn't depend on the dual used to to compute it, one can try to compute the Lefschetz invariant of a given morphism using two different duals and thereby get an identity in equivariant K-homology (see \$2). Such examples (worked out in [13] and [15]), seemed to us interesting enough to support making a systematic study of the Lefschetz map. However, to get started on this question one obviously has to first describe the morphisms $f$ themselves in some kind of satisfactory way. To this end, we have extended the theory of correspondences initiated by Baum, Connes, Skandalis and others, to the equivariant situation, in the paper [16]. This extension presents some new features, and we will devote some space in this survey to explaining them.

If $\mathcal{G}$ is a proper groupoid and $X$ and $Y$ are $\mathcal{G}$-spaces, then an equivariant correspondence from $X$ to $Y$ is a quadruple $(M, b, f, \xi)$ where $M$ is a $\mathcal{G}$-space, $b: M \rightarrow X$ is a $\mathcal{G}$-map (not necessarily proper), $\xi$ is an equivariant $\mathrm{K}$-theory class with compact vertical support along the fibres of $b$, and $f$ is a K-oriented normal map from $M$ to $Y$ (see Definition 3.11). For example, if $\mathcal{G}$ is a compact group, $Y$ is a point and $M$ is compact, then an normal map $M \rightarrow Y$ is the specification of an orthogonal representation of $\mathcal{G}$ on some $\mathbb{R}^{n}$, an equivariant vector bundle $V$ over $M$, and an open equivariant embedding $\hat{f}: V \rightarrow \mathbb{R}^{n}$. To construct an example of such a triple, assume that $M$ has been given the structure of a smooth manifold, and that $\mathcal{G}$ acts smoothly. In this case we may appeal to a theorem of Mostow to embed $M$ is a finite-dimensional linear representation of $\mathcal{G}$, then take $V$ to be the normal bundle to the embedding.

There is a topologically defined equivalence relation on correspondences that makes the set of equivalence classes of $\mathcal{G}$-equivariant correspondences from $X$ to $Y$ the morphism set $\widehat{\mathrm{KK}}_{*}^{\mathcal{G}}(X, Y)$ in a category $\widehat{\mathrm{KK}}^{\mathcal{G}}$ which maps naturally to $\mathrm{KK}^{\mathcal{G}}$. For example, let $\mathcal{G}$ be compact group and let both $X$ and $Y$ be the one-point space. Let $M$ be a smooth, compact, equivariantly $\mathrm{K}$-oriented $\mathcal{G}$-manifold, $\xi \in \mathrm{K}_{\mathcal{G}}^{0}(M)$ be an equivariant K-theory class for $M$ represented by an equivariant vector bundle $V$ on $M$. By embedding $M$ in a finite-dimensional representation of $\mathcal{G}$ as in the previous paragraph, we can endow the map from $M$ to $Y:=\star$ with the structure of a smooth, $\mathrm{K}$-oriented, normal map, and we obtain a $\mathcal{G}$-equivariant correspondence $(M, \star, \star, \xi)$ from a point to a point, and so a class in $\widehat{\mathrm{KK}}_{*}^{\mathcal{G}}(\star, \star)$. Applying the natural map $\widehat{\mathrm{KK}}_{*}^{\mathcal{G}}(\star, \star) \rightarrow \mathrm{KK}_{*}^{\mathcal{G}}(\mathbb{C}, \mathbb{C}) \cong \operatorname{Rep}(\mathcal{G})$ maps this correspondence to the $\mathcal{G}$-equivariant topological index of $D_{V}$ in the sense of [1], where $D_{V}$ is the Dirac operator on $M$ twisted by the equivariant vector bundle $V$. By the Atiyah-Singer Index theoreom, this of course agrees with the $\mathcal{G}$-equivariant analytic index of $D_{V}$ in $\operatorname{Rep}(\mathcal{G})$, obtained by considering the difference of finite-dimensional $\mathcal{G}$-representations on the kernel and cokernel of $D_{V}$.

The combination of the Atiyah-Singer index theorem and the theory of equivariant correspondences represents a powerful tool, because while the index theorem allows us to translate analytic problems into topological ones, the theory of correspondences allows us to manipulate this topological data in interesting ways. We will illustrate this in connection with the Borel-Bott-Weil theorem in $\$ 3.8$. Here the goal is to determine relations between the so-called holomorphic representations of a complex, semisimple Lie group $G$, obtained by letting $G$ act on appropriate Dolbeault cohomology groups. The problem can be reformulated in a rather obvious way in terms of equivariant index theory, that is in terms of equivariant KK-theory, 
at least if we replace in the discussion $G$ by its maximal compact subgroup, from the point of view of Kasparov theory involving no loss of generality because of the Baum-Connes conjecture for amenable groupoids. Fix a Cartan subgroup $H \subset G$. Choose any two minimal parabolics containing $H$. Associated to $H, P, Q$ are two families of representations of $G$. There is no equivariant map from $G / P$ to $G / Q$ in this situation which can be used to intertwine these families, but we show that there is an equivariant correspondence which does it - up to the transformation rule predicted by the Borel-Bott-Weil theorem. The key steps are thus the Atiyah-Singer index theorem, used to replace the representations by their topological models, and the construction of an appropriate correspondence.

We mainly discuss the Borel-Bott-Weil theorem because it gives a good example of the theory of equivariant correspondences. In terms of the Lefschetz map, the fact that correspondences can be composed in an essentially topological fashion has the consequence that the Lefshetz invariants of self-correspondences of a smooth $\mathcal{G}$-manifold $X$, or, or more precisely, of their images in $\mathrm{KK}^{\mathcal{G}}$, can be computed topologically in terms of considerations of transversality. We explain the outcome of this computation in Section 4. the gist is that the Lefschetz invariant of a smooth equivariant self-correspondence $\Psi$ of a smooth $\mathcal{G}$-manifold $X$ in general position, can be described in terms of a $\mathcal{G}$-space called the coincidence space of the correspondence: it inherits from the smooth structure and K-orientation on $\Psi$ the structure of a smooth and equivariantly $\mathrm{K}$-oriented $\mathcal{G}$-manifold $\mathrm{F}_{\Psi}$ which maps to $X$ and represents an equivariant correspondence from $X$ to $Z$, thus a cycle for $\widehat{\mathrm{KK}}_{\mathcal{G}}^{*}(X, Z)$ and then a class in $\mathrm{KK}_{*}^{\mathcal{G}}\left(\mathcal{C}_{0}(X), \mathcal{C}_{0}(Z)\right)$. See Theorem4.12 for the exact statement. For example, if $\mathcal{G}$ is a compact group, $X$ a smooth and compact manifold with a smooth action of $\mathcal{G}$, then the Lefschetz invariant of a smooth equivariant map $f: X \rightarrow X$ in general position is the fixed-point set of the map, which is a finite set of points permuted by $\mathcal{G}, \mathrm{K}$-oriented by an equivariant line bundle over this finite set. This bundle depends on orientation data from the original map $f$ in a manner which reduces to the classical choice of signs at each fixed-point, when $\mathcal{G}$ is trivial. Thus, the topological model of the Lefschetz map provided by the theory of correspondences reveals a fixed-point theory for correspondences.

One naturally asks when the map $\widehat{\mathrm{KK}}_{\mathcal{G}}(X, Y) \rightarrow \mathrm{KK}^{\mathcal{G}}\left(\mathcal{C}_{0}(X), \mathcal{C}_{0}(Y)\right)$ is an isomorphism. We explain our results on this in $\$ 4.1$ once again, they rely on duality in a crucial way. When they apply, the topological and analytic Lefschetz maps are equivalent.

What is duality? It is central to our whole framework, and accordingly we begin the article with a discussion of it. It is well-known from the work of Kasparov and Connes and Skandalis (see [20] and [8]) that if if $X$ is a smooth manifold, then there is a natural family of isomorphisms

$$
\mathrm{KK}\left(\mathcal{C}_{0}(\mathrm{~T} X), \mathbb{C}\right) \cong \mathrm{RK}^{*}(X):=\mathrm{KK}^{X}\left(\mathcal{C}_{0}(X), \mathcal{C}_{0}(X)\right)
$$

where the groupoid equivariant KK group on the right is representible K-theory, or K-theory with locally finite support, denoted $\mathrm{RK}^{*}(X)$ by Kasparov. There is a generalisation of this duality to the equivariant situation if $\mathcal{G}$ acts smoothly and properly on $X$, and furthermore, the roles of $X$ and $\mathrm{T} X$ can be in a sense reversed, so that one can establish a pair of natural (in a technical sense) familes of isomorphisms

$$
\mathrm{KK}_{*}^{\mathcal{G}}\left(\mathcal{C}_{0}(\mathrm{~T} X) \otimes A, B\right) \cong \mathrm{KK}_{*}^{\mathcal{G} \ltimes X}\left(\mathcal{C}_{0}(X) \otimes A, \mathcal{C}_{0}(X) \otimes B\right)
$$

and

$$
\mathrm{KK}_{*}^{\mathcal{G}}\left(\mathcal{C}_{0}(X) \otimes A, B\right) \cong \mathrm{KK}_{*}^{\mathcal{G} \ltimes X}\left(\mathcal{C}_{0}(X), \mathcal{C}_{0}(\mathrm{~T} X) \otimes B\right)
$$


for all $\mathcal{G}$-C ${ }^{*}$-algebras $A$ and $B$. (The tensor products are all in the category of $\mathcal{G}$ - $\mathrm{C}^{*}$-algebras.) These results are proved in [11]. It is the first kind of duality (0.2) which is relevant for the Lefschetz map, and the second (0.3) that is used to prove that the map $\widehat{\mathrm{KK}^{\mathcal{G}}} \rightarrow \mathrm{KK}^{\mathcal{G}}$ is an isomorphism in certain cases. The idea behind the latter argument is that since the duality isomorphisms (0.3) are themselves induced by equivariant correspondences, duality can be used simultaneously in both the analytic and topological categories to to reduce proving bijectivity of

$$
\widehat{\mathrm{KK}}^{\mathcal{G}}(X, Y) \rightarrow \mathrm{KK}^{\mathcal{G}}\left(\mathcal{C}_{0}(X), \mathcal{C}_{0}(Y)\right)
$$

to proving bijectivity of

$$
\widehat{\mathrm{KK}}^{\mathcal{G} \ltimes X}(X, \mathrm{~T} X) \rightarrow \mathrm{KK}^{\mathcal{G} \ltimes X}\left(\mathcal{C}_{0}(X), \mathcal{C}_{0}(\mathrm{~T} X)\right)=\mathrm{RK}_{\mathcal{G}, X}^{0}(\mathrm{~T} X) .
$$

What is needed to make this work is then a topological model of duality. The main new issue that appears is that our equivariant correspondences require a good supply of equivariant vector bundles and this forces conditions on the groupoid $\mathcal{G}$. These considerations have in fact already appeared in the literature in connection with the groupoids $\Gamma \ltimes \mathcal{E} \Gamma$ in work by Wolfang Lück and Bob Oliver in 22 (see $\$ 3.3$ for the details) where $\Gamma$ is a discrete group and $\mathcal{E} \Gamma$ is its classifying space for proper actions. We explain exactly what the conditions are and how they are related to embedding theorems generalizing the embedding theorem of Mostow alluded to above.

The classical Lefschetz fixed-point theorem relates fixed-points of a map and the homological invariant of the map obtained by taking the graded trace of the induced map on homology, called the Lefschetz number. We finish this survey by introducing some global, homological invariants of correspondences which generalize the Lefschetz number. Roughly speaking, a Kasparov self-morphism, and in particular a self-correspondence should be considered as determining a linear operator on homology instead of just a number. We call it the Lefschetz operator. We then extend the classical Lefschetz fixed-point theorem to correspondences by identifying the Lefschetz operator with the operator of pairing with the Lefschetz invariant. In situations where a local index formula is available, this results in a description of the Lefschetz operator in terms of integrals of appropriate characteristic classes.

All spaces $X, Y$ etc in this article are required to be paracompact and Hausdorff and locally compact.

\section{Abstract Duality And the Lefschetz maP}

Throughout this paper, groupoid shall mean locally compact Hausdorff groupoid with Haar system. All topological spaces will be assumed paracompact and Hausdorff. For the material in this section, see 11. For source material on equivariant KK-theory for groupoids, see 25.

Let $\mathcal{G}$ be a groupoid. We let $Z$ denote the base space. A $\mathcal{G}$-C ${ }^{*}$-algebra is a $\mathrm{C}^{*}$-algebra $A$ with a ${ }^{*}$-homomorphism from $\mathcal{C}_{0}(Z)$ to central multipliers of $A$. This endows $A$ with the structure of a bundle of $\mathrm{C}^{*}$-algebras over $Z$ with groupoid elements mapping between the fibres in the obvious way. We often use the notation $\mathbb{1}:=\mathcal{C}_{0}(Z)$ because $\mathcal{C}_{0}(Z)$ is the tensor unit in the tensor category of $\mathcal{G}$-C $\mathrm{C}^{*}$-algebras, with tensor product obtained by letting $A \otimes B$ be the restriction to the diagonal $Z \subset Z \times Z$ of the ordinary tensor product of $A$ and $B$, the latter being in a canonical way a $\mathrm{C}^{*}$-algebra over $Z \times Z$. If $\mathcal{G}$ acts on a space $X$ then we sometimes denote by $\mathbb{1}_{X}$ the $\mathcal{G}$-C $\mathrm{C}^{*}$-algebra $\mathcal{C}_{0}(X)$; thus $\mathbb{1}_{X}$ is the tensor unit in the category of $\mathcal{G} \ltimes X$ $\mathrm{C}^{*}$-algebras, $X$ being the base of $\mathcal{G} \ltimes X$. For more details on basic aspects of $\mathcal{G}$-C ${ }^{*}$-algebras, see the first chapter of 11 .

If $A$ and $B$ are $\mathcal{G}$-C ${ }^{*}$-algebras, then the group $\operatorname{RKK}^{\mathcal{G}}(X ; A, B)$ is by definition the groupoid-equivariant Kasparov group $\mathrm{KK}^{\mathcal{G} \ltimes X}\left(\mathbb{1}_{X} \otimes A, \mathbb{1}_{X} \otimes B\right)$. This group 
differs from $\mathrm{KK}^{\mathcal{G}}\left(A, \mathbb{1}_{X} \otimes B\right)$ only in the support condition on cycles. For example if $\mathcal{G}$ is trivial and $A=B=\mathbb{C}$ then $\mathrm{KK}^{\mathcal{G}}\left(A, \mathbb{1}_{X} \otimes B\right)$ is the ordinary K-theory of $X$ and $\operatorname{RKK}^{\mathcal{G}}(X ; A, B)$ is the representible K-theory of $X$ (a non-compactly supported theory.)

Thus, even if the reader is only interested in groups, or the trivial group, it is convenient to introduce groupoids to some extent in order to describe cohomology theories with different support conditions.

If $P$ is a $\mathcal{G} \ltimes X$-algebra, we denote by $T_{P}$ the map

$$
\operatorname{RKK}^{\mathcal{G}}(X ; A, B):=\mathrm{KK}^{\mathcal{G} \ltimes X}\left(\mathbb{1}_{X} \otimes A, \mathbb{1}_{X} \otimes B\right) \rightarrow \mathrm{KK}^{\mathcal{G}}(P \otimes A, P \otimes B)
$$

which sends a $\mathcal{G} \ltimes X$-equivariant right Hilbert $\mathbb{1}_{X} \otimes B$-Hilbert module $\mathcal{E}$ to $\mathcal{E} \otimes P$, the tensor product being in the category of $\mathcal{G} \ltimes X$-algebras, and sends $F \in \mathbb{B}(\mathcal{E})$ to the operator $F \otimes \mathrm{id}_{P}$. This makes sense since $F$ commutes with the $\mathcal{C}_{0}(X)$-structure on $\mathcal{E}$. Note that this functor is the composition of external product

$$
\sqcup \otimes_{X} 1_{P}: \mathrm{KK}^{\mathcal{G} \ltimes X}\left(\mathbb{1}_{X} \otimes A, \mathbb{1}_{X} \otimes B\right) \rightarrow \mathrm{KK}^{\mathcal{G} \ltimes X}(A \otimes P, B \otimes P)
$$

(where the $X$-structure on $A \otimes P$ etc. is on the $P$ factor), and the forgetful map

$$
\mathrm{KK}^{\mathcal{G} \ltimes X}(A \otimes P, B \otimes P) \rightarrow \mathrm{KK}^{\mathcal{G}}(A \otimes P, B \otimes P)
$$

which forgets the $X$-structure.

1.1. Kasparov duals. We start by formalizing some duality calculations of Kasparov, c.f. [20, Theorem 4.9]. Explicit examples will be discussed later.

Definition 1.1. Let $n \in \mathbb{Z}$. An $n$-dimensional $\mathcal{G}$-equivariant Kasparov dual for the $\mathcal{G}$-space $X$ is a triple $(P, D, \Theta)$, where

- $P$ is a (possibly $\mathbb{Z} / 2$-graded) $\mathcal{G} \ltimes X$-C $\mathrm{C}^{*}$-algebra,

- $D \in \mathrm{KK}_{-n}^{\mathcal{G}}(P, \mathbb{1})$, and

- $\Theta \in \operatorname{RKK}_{n}^{\mathcal{G}}(X ; 1, P)$,

subject to the following conditions:

(1) $\Theta \otimes_{P} D=\operatorname{id}_{\mathbb{1}}$ in $\operatorname{RKK}_{0}^{\mathcal{G}}(X ; \mathbb{1}, \mathbb{1})$;

(2) $\Theta \otimes f=\Theta \otimes_{P} T_{P}(f)$ in $\operatorname{RKK}_{*+n}^{\mathcal{G}}(X ; A, B \otimes P)$ for all $\mathcal{G}$-C ${ }^{*}$-algebras $A$ and $B$ and all $f \in \mathrm{RKK}_{*}^{\mathcal{G}}(X ; A, B)$;

(3) $T_{P}(\Theta) \otimes_{P \otimes P} \Phi_{P}=(-1)^{n} T_{P}(\Theta)$ in $\operatorname{KK}_{n}^{\mathcal{G}}(P, P \otimes P)$, where $\Phi_{P}$ is the flip automorphism on $P \otimes P$.

The following theorem is proved in [1].

Theorem 1.2. Let $n \in \mathbb{Z}$, let $P$ be a $\mathcal{G} \ltimes X-\mathrm{C}^{*}$-algebra, $D \in \mathrm{KK}_{-n}^{\mathcal{G}}(P, \mathbb{1})$, and $\Theta \in \operatorname{RKK}_{n}^{\mathcal{G}}(X ; \mathbb{1}, P)$. Define two natural transformations

$$
\begin{aligned}
\mathrm{PD}: \mathrm{KK}_{i-n}^{\mathcal{G}}(P \otimes A, B) \rightarrow \operatorname{RKK}_{i}^{\mathcal{G}}(X ; A, B), & f \mapsto \Theta \otimes_{P} f, \\
\mathrm{PD}^{*}: \operatorname{RKK}_{i}^{\mathcal{G}}(X ; A, B) \rightarrow \mathrm{KK}_{i-n}^{\mathcal{G}}(P \otimes A, B), & g \mapsto(-1)^{i n} T_{P}(g) \otimes_{P} D,
\end{aligned}
$$

These two are inverse to each other if and only if $(P, D, \Theta)$ is an $n$-dimensional $\mathcal{G}$-equivariant Kasparov dual for $X$.

1.2. Abstract duals. The reader may have noticed that the only place the $\mathcal{C}_{0}(X)$ structure on $P$ comes into play in the conditions listed in Definition 1.1, and in the statement of Theorem [1.2, is via the functor $T_{P}$. In particular, if one has a Kasparov dual $(P, D, \Theta)$ and if one changes the $\mathcal{C}_{0}(X)$-structure on $P$, for example by composing it with a $\mathcal{G}$-equivariant homeomorphism of $X$, then the map PD of Theorem 1.2 does not change; since by the theorem $\mathrm{PD}^{*}$ is its inverse map, it would not change either, strangely, since its definition uses $T_{P}$. In fact it turns out that the functor $T_{P}$ can be reconstructed from $\mathrm{PD}$ if one knows that $\mathrm{PD}$ is an 
isomorphism. This is an important idea in connection with the Lefschetz map and suggests the following useful definition.

Definition 1.3. An $n$-dimensional abstract dual for $X$ is a pair $(P, \Theta)$, where $P$ is a $\mathcal{G}$-C $\mathrm{C}^{*}$-algebra and $\Theta \in \operatorname{RKK}_{n}^{\mathcal{G}}(X ; \mathbb{1}, P)$, such that the map PD defined as in Theorem 1.2 is an isomorphism for all $\mathcal{G}$ - $\mathrm{C}^{*}$-algebras $A$ and $B$.

This definition is shorter, and, as mentioned, is useful for theoretical reasons, but it seems like it should be difficult to check in practise.

In any case, it is clear from Theorem 1.2 that a pair $(P, \Theta)$ is an abstract dual if it is part of a Kasparov dual $(P, D, \Theta)$.

Proposition 1.4. An abstract dual for a space $X$ is unique up to a canonical $\mathrm{KK}^{\mathcal{G}}$-equivalence if it exists, and even covariantly functorial in the following sense.

Let $X$ and $Y$ be two $\mathcal{G}$-spaces and let $f: X \rightarrow Y$ be a $\mathcal{G}$-equivariant continuous map. Let $\left(P_{X}, \Theta_{X}\right)$ and $\left(P_{Y}, \Theta_{Y}\right)$ be abstract duals for $X$ and $Y$ of dimensions $n_{X}$ and $n_{Y}$, respectively. Then there is a unique $P_{f} \in \mathrm{KK}_{n_{Y}-n_{X}}^{\mathcal{G}}\left(P_{X}, P_{Y}\right)$ with $\Theta_{X} \otimes_{P_{X}} P_{f}=f^{*}\left(\Theta_{Y}\right)$. Given two composable maps between three spaces with duals, we have $P_{f \circ g}=P_{f} \circ P_{g}$. If $X=Y, f=\operatorname{id}_{X}$, and $\left(P_{X}, \Theta_{X}\right)=\left(P_{Y}, \Theta_{Y}\right)$, then $P_{f}=\mathrm{id}_{P_{X}}$. If only $X=Y, f=\mathrm{id}_{X}$, then $P_{f}$ is a $\mathrm{KK}^{\mathcal{G}}$-equivalence between the two duals of $X$.

Although the map $f: X \rightarrow Y$ appearing in Proposition 1.4 does not have to be proper, it nonetheless yields a morphism $P_{f}$ in $\mathrm{KK}^{\mathcal{G}}$.

1.3. Duality co-algebra. Let $(P, \Theta)$ be an $n$-dimensional abstract dual for a $\mathcal{G}$-space $X$. By the Yoneda Lemma, another abstract dual $\left(P^{\prime}, \Theta^{\prime}\right)$ also for $X$ and say of dimension $n^{\prime}$ is related to $(P, \Theta)$ by an invertible element

$$
\psi \in \mathrm{KK}_{n^{\prime}-n}^{\mathcal{G}}\left(P, P^{\prime}\right) \text {, such that } \Theta \otimes_{P} \psi=\Theta^{\prime} .
$$

We repeat for emphasis that since $(P, \Theta)$ is only an abstract dual, we are not assuming that there is a $\mathcal{G} \ltimes X$-structure on $P$. However, we are going to attempt to reconstruct what we might consider to be a $\mathcal{G} \ltimes X$-structure on $P$ at the level of KK-theory. Along the way we will keep track of how the change in dual from $(P, \Theta)$ to $\left(P^{\prime}, \Theta^{\prime}\right)$ affects our constructions.

Define $D \in \mathrm{KK}_{-n}^{\mathcal{G}}(P, \mathbb{1})$ by the requirement

$$
\operatorname{PD}(D):=\Theta \otimes_{P} D=1_{\mathbb{1}} \quad \text { in } \operatorname{RKK}_{0}^{\mathcal{G}}(X ; \mathbb{1}, \mathbb{1}) .
$$

as in the first condition in Definition 1.1). The class $D$ should thus play the role of the class named $D$ in a Kasparov dual. It is routine to check that when we change the dual, as above, $D$ is replaced by $\psi^{-1} \otimes_{P} D$.

We call $D$ counit of the duality because it plays the algebraic role of a counit in the theory of adjoint functors (see [1] and also Remark 3.2 below).

Define $\nabla \in \mathrm{KK}_{n}^{\mathcal{G}}(P, P \otimes P)$ by the requirement that

$$
\operatorname{PD}(\nabla):=\Theta \otimes_{P} \nabla=\Theta \otimes_{X} \Theta \quad \text { in } \operatorname{RKK}_{2 n}^{\mathcal{G}}(X ; \mathbb{1}, P \otimes P) .
$$

We call $\nabla$ the comultiplication of the duality. When we change the dual, $\nabla$ is replaced by

$$
(-1)^{n\left(n^{\prime}-n\right)} \psi^{-1} \otimes_{P} \nabla \otimes_{P \otimes P}(\psi \otimes \psi) \in \mathrm{KK}_{n^{\prime}}^{\mathcal{G}}\left(P^{\prime}, P^{\prime} \otimes P^{\prime}\right) .
$$

Remark 1.7. If $n=0$ then the object $P$ of $\mathrm{KK}^{\mathcal{G}}$ with counit $D$ and comultiplication $\nabla$ is a cocommutative, counital coalgebra object in the tensor category $\mathrm{KK}^{\mathcal{G}}$ :

$$
\begin{gathered}
\nabla \otimes_{P \otimes P}\left(\nabla \otimes 1_{P}\right)=\nabla \otimes_{P \otimes P}\left(1_{P} \otimes \nabla\right), \\
\nabla \otimes_{P \otimes P} \Phi_{P}=\nabla, \\
\nabla \otimes_{P \otimes P}\left(D \otimes 1_{P}\right)=1_{P}=\nabla \otimes_{P \otimes P}\left(1_{P} \otimes D\right) .
\end{gathered}
$$


Equation (1.8) holds in $\mathrm{KK}_{2 n}^{\mathcal{G}}\left(P, P^{\otimes 3}\right)$, equation (1.9) holds in $\mathrm{KK}_{n}^{\mathcal{G}}(P, P \otimes P)$, and (1.10) holds in $\mathrm{KK}_{0}^{\mathcal{G}}(P, P)$.

Now, for $\mathcal{G}$ - $\mathrm{C}^{*}$-algebras $A$ and $B$, we define

$$
T_{P}^{\prime}: \operatorname{RKK}_{*}^{\mathcal{G}}(X ; A, B) \rightarrow \mathrm{KK}_{*}^{\mathcal{G}}(P \otimes A, P \otimes B), \quad f \mapsto \nabla \otimes_{P} \mathrm{PD}^{-1}(f),
$$

where PD is the duality isomorphism, $\nabla$ is the comultiplication of the duality, and $\otimes_{P}$ operates on the second copy of $P$ in the target $P \otimes P$ of $\nabla$. A computation yields that

$$
\operatorname{PD}\left(T_{P}^{\prime}(f)\right)=\Theta \otimes_{X} f \quad \text { in } \operatorname{RKK}_{i+n}^{\mathcal{G}}(X ; A, P \otimes B)
$$

for all $f \in \operatorname{RKK}_{i}^{\mathcal{G}}(X ; A, B)$. It follows that

$$
T_{P}^{\prime}(f)=T_{P}(f)
$$

if $(P, \Theta)$ is part of a Kasparov dual, and thus $T_{P}$ is in fact independent of the $\mathcal{G} \ltimes X$-structure on $P$, verifying our guess above.

When we change the dual, we replace $T_{P}^{\prime}$ by the map

$$
\operatorname{RKK}_{i}^{\mathcal{G}}(X ; A, B) \ni f \mapsto(-1)^{i\left(n-n^{\prime}\right)} \psi^{-1} \otimes_{P} T_{P}(f) \otimes_{P} \psi \in \mathrm{KK}_{i}^{\mathcal{G}}\left(P^{\prime} \otimes A, P^{\prime} \otimes B\right) .
$$

In fact, one can check that the maps $T_{P}^{\prime}$ above define a functor

$$
T_{P}^{\prime}: \operatorname{RKK}^{\mathcal{G}}(X) \rightarrow \mathrm{KK}^{\mathcal{G}}
$$

This is a $\mathrm{KK}^{\mathcal{G}}$-functor in the sense that it is compatible with the tensor products $\otimes$, and it is left adjoint to the functor $p_{X}^{*}: \mathrm{KK}^{\mathcal{G}} \rightarrow \mathrm{RKK}^{\mathcal{G}}$ induced from the groupoid homomorphism $\mathcal{G} \ltimes X \rightarrow \mathcal{G}$.

It follows that we can write the inverse duality map involved in an abstract dual $(P, \Theta)$ as:

$$
\mathrm{PD}^{-1}(f)=(-1)^{i n} T_{P}^{\prime}(f) \otimes_{P} D \quad \text { in } \mathrm{KK}_{i-n}^{\mathcal{G}}(P \otimes A, B)
$$

for $f \in \operatorname{RKK}_{i}^{\mathcal{G}}(X ; A, B)$. By the above discussion this formula agrees with the map $\mathrm{PD}^{*}$ when we have a Kasparov dual.

1.4. The Lefschetz map. The formal computations summarized in the previous section allows us to single out an interesting invariant of a $\mathcal{G}$-space $X$, at least under the hypothesis that $X$ has some abstract dual.

For any $\mathcal{G}$-space $X$ the diagonal embedding $X \rightarrow X \times_{Z} X$ is a proper map and hence induces a *-homomorphism

$$
\mathbb{1}_{X} \otimes \mathbb{1}_{X} \cong \mathcal{C}_{0}\left(X \times_{Z} X\right) \rightarrow \mathcal{C}_{0}(X)=\mathbb{1}_{X}
$$

This map is $\mathcal{G} \ltimes X$-equivariant and hence yields

$$
\Delta_{X} \in \operatorname{RKK}^{\mathcal{G}}\left(X ; \mathbb{1}_{X}, \mathbb{1}\right) \cong \mathrm{KK}^{\mathcal{G} \ltimes X}\left(\mathcal{C}_{0}\left(X \times_{Z} X\right), \mathcal{C}_{0}(X)\right) .
$$

We call this the diagonal restriction class. It yields a canonical map

$$
\sqcup \otimes_{\mathbb{1}_{X}} \Delta_{X}: \mathrm{KK}^{\mathcal{G}}\left(\mathbb{1}_{X} \otimes A, \mathbb{1}_{X} \otimes B\right) \rightarrow \operatorname{RKK}^{\mathcal{G}}\left(X ; \mathbb{1}_{X} \otimes A, B\right) .
$$

In particular, this contains a map $\operatorname{KK}^{\mathcal{G}}\left(\mathbb{1}_{X}, \mathbb{1}_{X}\right) \rightarrow \operatorname{RKK}^{\mathcal{G}}\left(X ; \mathbb{1}_{X}, \mathbb{1}\right)$.

Example 1.15. If $f: X \rightarrow X$ is a proper, continuous, $\mathcal{G}$-equivariant map, then

$$
[f] \otimes_{\mathbb{1}_{X}} \Delta_{X} \in \operatorname{RKK}^{\mathcal{G}}\left(X ; \mathbb{1}_{X}, \mathbb{1}\right)
$$

is the class of the ${ }^{*}$-homomorphism induced by $\left(\operatorname{id}_{X}, f\right): X \rightarrow X \times_{Z} X$.

Now drop the assumption that $f$ be proper. Then $\left(\operatorname{id}_{X}, f\right)$ is still a proper, continuous, $\mathcal{G}$-equivariant map. The class of the ${ }^{*}$-homomorphism it induces is equal to $f^{*}\left(\Delta_{X}\right)$, where we use the maps

$$
f^{*}: \operatorname{RKK}_{*}^{\mathcal{G}}(X ; A, B) \rightarrow \operatorname{RKK}_{*}^{\mathcal{G}}(X ; A, B)
$$


for $A=\mathbb{1}_{X}, B=\mathbb{1}$ induced by $f: X \rightarrow X$ (the functor $X \mapsto \operatorname{RKK}^{\mathcal{G}}(X ; A, B)$ is functorial with respect to arbitrary $\mathcal{G}$-maps, not just proper ones.) This suggests that we can think of $\operatorname{RKK}^{\mathcal{G}}\left(X ; \mathbb{1}_{X}, \mathbb{1}\right)$ as generalized, possibly non-proper self-maps of $X$.

In fact if the anchor map $X \rightarrow Z$ is a proper map, so that $X$ is a bundle of compact spaces over $Z$, then $\sqcup \otimes_{\mathbb{1}_{X}} \Delta_{X}$ is an isomophism (an easy exercise in the definitions.)

Now let $T_{P}^{\prime}$ be the tensor functor and $\Delta_{X}$ the diagonal restriction class of an abstract dual. We define the multiplication class of $P$ by

$$
[m]:=T_{P}^{\prime}\left(\Delta_{X}\right) \in \mathrm{KK}_{0}^{\mathcal{G}}\left(P \otimes \mathbb{1}_{X}, P\right) .
$$

A change of dual as in (1.5) replaces $[m]$ by $\psi^{-1} \otimes_{P}[m] \otimes_{P} \psi$.

Lemma 1.17. Let $(P, D, \Theta)$ be a Kasparov dual. Then $[m]$ is the class in $\mathrm{KK}^{\mathcal{G}}$ of the multiplication homomorphism $\mathcal{C}_{0}(X) \otimes_{Z} P \rightarrow P$ that describes the $X$-structure on $P$ (up to commuting the tensor factors).

We now have enough theoretical development to define the Lefschetz map and sketch the proof of its homotopy invariance.

Let $X$ be a $\mathcal{G}$-space and $(P, \Theta)$ an $n$-dimensional abstract dual for $X, \mathrm{PD}$ and $\mathrm{PD}^{-1}$ the duality isomorphisms. As before, we write $\mathbb{1}:=\mathcal{C}_{0}(Z), \mathbb{1}_{X}:=\mathcal{C}_{0}(X)$ and $\Delta_{X} \in \operatorname{RKK}^{\mathcal{G}}\left(X ; \mathbb{1}_{X}, \mathbb{1}\right)=\mathrm{KK}^{\mathcal{G} \ltimes X}\left(\mathbb{1}_{X} \otimes \mathbb{1}_{X}, \mathbb{1}_{X}\right)$ the diagonal restriction class and

$$
\bar{\Theta}:=\operatorname{forget}_{X}(\Theta) \in \mathrm{KK}_{n}^{\mathcal{G}}\left(\mathbb{1}_{X}, P \otimes \mathbb{1}_{X}\right) .
$$

Definition 1.18. The equivariant Lefschetz map

$$
\text { Lef : } \operatorname{RKK}_{*}^{\mathcal{G}}\left(X ; \mathbb{1}_{X}, \mathbb{1}\right) \rightarrow \mathrm{KK}_{*}^{\mathcal{G}}\left(\mathbb{1}_{X}, \mathbb{1}\right)
$$

for a $\mathcal{G}$-space $X$ is defined as the composite map

$$
\operatorname{RKK}_{i}^{\mathcal{G}}\left(X ; \mathbb{1}_{X}, \mathbb{1}\right) \stackrel{\mathrm{PD}^{-1}}{\longrightarrow} \mathrm{KK}_{i-n}^{\mathcal{G}}\left(P \otimes \mathbb{1}_{X}, \mathbb{1}\right) \stackrel{\bar{\Theta} \otimes_{P \otimes 1} \sqcup}{\longrightarrow} \mathrm{KK}_{i}^{\mathcal{G}}\left(\mathbb{1}_{X}, \mathbb{1}\right) .
$$

The equivariant Euler characteristic of $X$ is

$$
\operatorname{Eul}_{X}:=\operatorname{Lef}\left(\Delta_{X}\right) \in \operatorname{KK}_{0}^{\mathcal{G}}\left(\mathbb{1}_{X}, \mathbb{1}\right)=\operatorname{KK}_{0}^{\mathcal{G}}\left(\mathcal{C}_{0}(X), \mathcal{C}_{0}(Z)\right)
$$

Let $f \in \operatorname{RKK}_{i}^{\mathcal{G}}\left(X ; \mathbb{1}_{X}, \mathbb{1}\right)$. Equations (1.13) and (1.16) yield

$$
\begin{aligned}
\operatorname{Lef}(f) & =(-1)^{i n} \bar{\Theta} \otimes_{P \otimes_{X}} T_{P}^{\prime}(f) \otimes_{P} D, \\
\operatorname{Eul}_{X} & =(-1)^{i n} \bar{\Theta} \otimes_{P \mathbb{1}_{X}}[m] \otimes_{P} D .
\end{aligned}
$$

We have already established in Lemma ?? that if $(P, \Theta)$ is part of a Kasparov dual, then $T_{P}^{\prime}=T_{P}$ and $[m]$ is the KK-class of the multiplication *-homomorphism $\mathcal{C}_{0}(X, P) \rightarrow P$, so that (1.19) yields explicit formulas for $\operatorname{Lef}(f)$ and $\operatorname{Eul}_{X}$. This is extremely important because otherwise it would not be possible to compute these invariants.

Let $X$ and $X^{\prime}$ be $\mathcal{G}$-spaces, and let $f: X \rightarrow X^{\prime}$ be a $\mathcal{G}$-homotopy equivalence. Then $f$ induces an equivalence of categories $\operatorname{RKK}^{\mathcal{G}}\left(X^{\prime}\right) \cong \operatorname{RKK}^{\mathcal{G}}(X)$, that is, we get invertible maps

$$
f^{*}: \operatorname{RKK}_{*}^{\mathcal{G}}\left(X^{\prime} ; A, B\right) \rightarrow \operatorname{RKK}_{*}^{\mathcal{G}}(X ; A, B)
$$

for all $\mathcal{G}$-C ${ }^{*}$-algebras $A$ and $B$. Now assume, in addition, that $f$ is proper; we do not need the inverse map or the homotopies to be proper. Then $f$ induces a *-homomorphism $f^{!}: \mathcal{C}_{0}\left(X^{\prime}\right) \rightarrow \mathcal{C}_{0}(X)$, which yields $\left[f^{!}\right] \in \mathrm{KK}^{\mathcal{G}}\left(\mathcal{C}_{0}\left(X^{\prime}\right), \mathcal{C}_{0}(X)\right)$. We write $\left[f^{!}\right]$instead of $\left[f^{*}\right]$ to better distinguish this from the map $f^{*}$ above. Unless $f$ is a proper $\mathcal{G}$-homotopy equivalence, $\left[f^{!}\right]$need not be invertible. 
Theorem 1.21. Let $X$ and $X^{\prime}$ be $\mathcal{G}$-spaces with abstract duals, and let $f: X \rightarrow X^{\prime}$ be both a proper map and a $\mathcal{G}$-homotopy equivalence. Then

$$
\left[f^{!}\right] \otimes_{\mathcal{C}_{0}(X)} \operatorname{Eul}_{X}=\operatorname{Eul}_{X^{\prime}} \quad \text { in } \operatorname{KK}_{0}^{\mathcal{G}}\left(\mathcal{C}_{0}\left(X^{\prime}\right), \mathbb{1}\right)
$$

and the Lefschetz maps for $X$ and $X^{\prime}$ are related by a commuting diagram

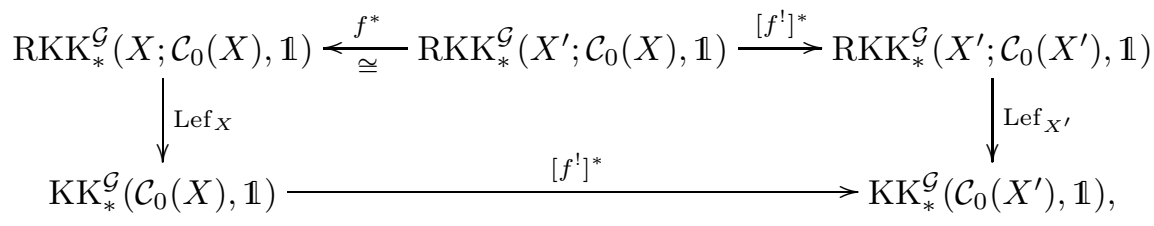

where $\left[f^{!}\right]^{*}$ denotes composition with $\left[f^{!}\right]$.

In particular, Eul $_{X}$ and the map Lef L $_{X}$ do not depend on the chosen dual.

The proof relies on the discussion preceding the theorem.

Theorem 1.21 implies that the Lefschetz maps for properly $\mathcal{G}$-homotopy equivalent spaces are equivalent because then $\left[f^{!}\right]$is invertible, so that all horizontal maps in the diagram in Theorem 1.21 are invertible. In this sense, the Lefschetz map and the Euler class are invariants of the proper $\mathcal{G}$-homotopy type of $X$.

The construction in Example 1.15 associates a class $\left[\Delta_{f}\right] \in \operatorname{RKK}_{0}^{\mathcal{G}}\left(X ; \mathcal{C}_{0}(X), \mathbb{1}\right)$ to any continuous, $\mathcal{G}$-equivariant map $f: X \rightarrow X$; it does not matter whether $f$ is proper. We abbreviate

$$
\operatorname{Lef}(f):=\operatorname{Lef}\left(\left[\Delta_{f}\right]\right)
$$

and call this the Lefschetz invariant of $f$. Of course, equivariantly homotopic selfmaps induce the same class in $\operatorname{RKK}^{\mathcal{G}}\left(X ; \mathcal{C}_{0}(X), \mathbb{1}\right)$ and therefore have the same Lefschetz invariant. We have $\operatorname{Lef}\left(\operatorname{id}_{X}\right)=\operatorname{Eul}_{X}$.

More generally, specializing (1.14) gives a map

$$
\sqcup \otimes_{\mathbb{1}_{X}} \Delta_{X}: \operatorname{KK}_{*}^{\mathcal{G}}\left(\mathcal{C}_{0}(X), \mathcal{C}_{0}(X)\right) \rightarrow \operatorname{RKK}_{*}^{\mathcal{G}}\left(X ; \mathcal{C}_{0}(X), \mathbb{1}\right),
$$

which we compose with the Lefschetz map; abusing notation, we still denote this composition by

$$
\text { Lef }: \mathrm{KK}_{*}^{\mathcal{G}}\left(\mathcal{C}_{0}(X), \mathcal{C}_{0}(X)\right) \rightarrow \mathrm{KK}_{*}^{\mathcal{G}}\left(\mathcal{C}_{0}(X), \mathbb{1}\right)
$$

Finally, we record that Lefschetz invariants for elements of $\operatorname{RKK}_{*}^{\mathcal{G}}\left(X ; \mathcal{C}_{0}(X), \mathbb{1}\right)$ can be arbitrarily complicated: the Lefschetz map is rather easily seen to be split surjective. The splitting is given by specializing the inflation map

$$
p_{X}^{*}: \mathrm{KK}_{*}^{\mathcal{G}}(A, B) \rightarrow \mathrm{KK}^{\mathcal{G} \ltimes X}\left(\mathbb{1}_{X} \otimes A, \mathbb{1}_{X} \otimes B\right)
$$

to $A:=\mathbb{1}_{X}$ and $B:=\mathbb{1}$.

The fundamental example of a Kasparov dual is provided by the vertical tangent space to a bundle of smooth manifolds over the base $Z$ of a groupoid, in which morphisms act smoothly. We come back to this in \$4. Rather than studying examples of duals right away, we give some examples of computations of the Lefschetz map in some relatively simple situations.

\section{Examples of COMputations of the Lefschetz map}

In this section we will give some examples of computations of the Lefschetz map for various instances of spaces with duals and for equivariant self-morphisms coming from actual maps. The problem of computing the Lefschetz invariants of more general Kasparov self-morphisms for the next section is a central problem for us, and will be treated in \$4 once we have available the theory of equivariant correspondences. 
Most of the examples are quite close to proper actions, but they do not quite have to be proper. The point is that an abstract (or Kasparov) dual for a $\mathcal{G}$-space $X$ also yields one for $X$ regarded as a $\mathcal{G}^{\prime}$-space where $\mathcal{G}^{\prime}$ is a (not necessarily closed) subgroupoid of $\mathcal{G}$. Even if $X$ is proper as a $\mathcal{G}$-space, it need not be proper as a $\mathcal{G}^{\prime}$-space since $\mathcal{G}^{\prime}$ may not be closed.

Known examples of duals are of two types; they are both Kasparov duals. If $\mathcal{G}$ is a locally compact group acting as simplicial automorphisms of a finite-dimensional simplicial complex then $X$ has a Kasparov dual by [13]. This does not quite imply that the action of $\mathcal{G}$ is proper, since the action of $\mathcal{G}$ could be trivial. Neither does it quite imply that $\mathcal{G}$ must be discrete, though since the connected component of the identity of $\mathcal{G}$ must act trivially, the only interesting examples must involve disconnected groups. For instance, the non-discrete group $\operatorname{SL}\left(2, \mathbb{Q}_{p}\right)$ acts simplicially on a tree.

If $X$ is a complete complete Riemannian manifold then $X$ is a proper $\mathcal{G}$-space where $\mathcal{G}$ is the Lie group of isometries of $X$, and either the Clifford algebra of $X$ or the $\mathrm{C}^{*}$-algebra of $\mathcal{C}_{0}$-functions on the tangent bundle $\mathrm{T} X$ of $X$ is part of a Kasparov dual for $X$ (see e.g. 13 or [11), and also \$4. Hence if $\mathcal{G}$ is any group of isometries of a Riemannian manifold $X$ with finitely many components, then the $\mathcal{G}$-space $X$ also has a Kasparov dual. If the tangent bundle TX admits an equivariant $\mathrm{K}$-orientation, then $\mathcal{C}_{0}(\mathrm{~T} X)$ can be replaced by $\mathcal{C}_{0}(X)$. For instance, the circle with the group $\mathbb{Z}$ acting by an irrational rotation has a Kasparov dual of of dimension 1 , given by $(\mathcal{C}(\mathbb{T}), D, \Theta)$ where $D$ is the Dirac operator on the circle.

The Lefschetz invariants of equivariant self-maps of $X$ will in both these situations turn out to be in some sense zero-dimensional in the sense that they are built out of point-evaluation classes. As we will see later, the Lefschetz invariants of more general Kasparov self-morphisms are more complicated objects.

2.1. The combinatorial Lefschetz map. Let $X$ be a finite-dimensional simplicial complex and let $G$ be a locally compact group acting smoothly and simplicially on $X$ (that is, stabilisers of points are open). We follow [13] and 15. Assume that $X$ admits a colouring (that is, $X$ is typed) and that $G$ preserves the colouring. This ensures that if $g \in G$ maps a simplex to itself, then it fixes that simplex pointwise.

Let $S X$ be the set of (non-degenerate) simplices of $X$ and let $S_{d} X \subseteq S X$ be the subset of $d$-dimensional simplices. The group $G$ acts on the discrete set $S X$ preserving the decomposition $S X=\bigsqcup S_{d} X$. Decompose $S X$ into $G$-orbits. For each orbit $\dot{\sigma} \subseteq S X$, choose a representative $\sigma \in S X$ and let $\xi_{\sigma} \in X$ be its barycentre and $\operatorname{Stab}(\sigma) \subseteq G$ its stabiliser. Restriction to the orbit $\dot{\sigma}$ defines a $G$-equivariant *-homomorphism

$$
\xi_{\dot{\sigma}}: \mathcal{C}_{0}(X) \rightarrow \mathcal{C}_{0}(G / \operatorname{Stab}(\sigma)) \rightarrow \mathbb{K}\left(\ell^{2}(G / \operatorname{Stab} \sigma)\right),
$$

where the second map is the representation by pointwise multiplication operators. We let $\left[\xi_{\dot{\sigma}}\right]$ be its class in $\mathrm{KK}_{0}^{G}\left(\mathcal{C}_{0}(X), \mathbb{1}\right)$.

Let $\gamma: X \rightarrow X$ be a $G$-equivariant self-map of $X$. Since $\gamma$ is $G$-equivariantly homotopic to a $G$-equivariant cellular map, we may assume without loss of generality that $\gamma$ is itself cellular. Hence it induces a $G$-equivariant chain map

$$
\gamma: C_{\bullet}(X) \rightarrow C_{\bullet}(X),
$$

where $C_{\bullet}(X)$ is the chain complex of oriented simplices of $X$. A basis for $C_{\bullet}(X)$ is given by the set of (un-)oriented simplices, by arbitrarily choosing an orientation on each simplex. We may describe the chain map $\gamma$ by its matrix coefficients $\Gamma_{\sigma \tau} \in \mathbb{Z}$ with respect to this basis; thus the subscripts are unoriented simplices. For example, 
if $\Gamma$ maps a simplex to itself, and reverses orientation, then $\Gamma_{\sigma, \sigma}=-1$. Since $\Gamma$ is $G$-equivariant, $\Gamma_{g(\sigma), g(\sigma)}=\Gamma_{\sigma \sigma}$. So the following makes sense.

Notation 2.2. For $\dot{\sigma} \in G \backslash S_{d} X$, let $\mathbf{n}(\Gamma, \dot{\sigma}):=(-1)^{d} \Gamma_{\sigma \sigma} \in \mathbb{Z}$ for any choice of representative $\sigma \in \dot{\sigma}$.

The following theorem is proved in [15] using the simplicial dual developed in [13, and inspired by ideas of Kasparov and Skandalis in [21].

Theorem 2.3. Let $X$ be a finite-dimensional coloured simplicial complex and let $G$ be a locally compact group that acts smoothly and simplicially on $X$, preserving the colouring. Let $\gamma: X \rightarrow X$ be a G-equivariant self-map. Define $\mathbf{n}(\Gamma, \dot{\sigma}) \in \mathbb{Z}$ and $\left[\xi_{\dot{\sigma}}\right] \in \mathrm{KK}^{G}\left(\mathcal{C}_{0}(X), \mathbb{1}\right)$ for $\dot{\sigma} \in G \backslash S X$ as above. Then

$$
\operatorname{Lef}(\gamma)=\sum_{\dot{\sigma} \in G \backslash S X} \mathbf{n}(\Gamma, \dot{\sigma})\left[\xi_{\dot{\sigma}}\right]
$$

In the non-equivariant situation, if $X$ is connected and has only a finite number of simplices, then the formula just given reduces to the ordinary Lefschetz number of $\gamma$ by the (standard) argument that proves that the Euler characteristic of a finite simplicial complex can be computed either by counting ranks of simplicial homology groups, or by directly counting the number of simplices in the complex. In the noncompact case this doesn't make sense anymore since the number of orbits of simplices may be infinite, but our definition of the Lefschetz invariant still makes sense. This illustrates the advantage of considering K-homology classes instead of numbers as fixed-point data.

2.2. The smooth Lefschetz map. For another example, let $X$ be a smooth manifold and $G$ a group acting by isometric diffeomorphisms of $X$. Then we can build a Kasparov dual for $X$ using the Clifford algebra. The real (resp. complex) Clifford algebra of a Euclidean vector space $V$ is generated as a real (resp. complex) unital * algebra by an orthonormal basis $\left\{e_{i}\right\}$ for $V$ with the relations that $e_{i}$ is self-adjoint and $e_{i} e_{j}+e_{j} e_{i}=2 \delta_{i j}$. In the complex case this produces a finite-dimensional $\mathbb{Z} / 2$-graded $\mathrm{C}^{*}$-algebra. More generally if $V$ is a Euclidean vector bundle, this construction applies and produces a locally trivial bundle of finite-dimensional $\mathbb{Z} / 2$ graded $\mathrm{C}^{*}$-algebras over $X$. If $V:=\mathrm{T} X$ for a Riemannian manifold $X$ then the Clifford algebra of $X$ is the corresponding $\mathrm{C}^{*}$-algebra of sections vanishing at infinity. It is denoted $\mathscr{C}_{\tau}(X)$. This $\mathrm{C}^{*}$-algebra carries a canonical action of the group of isometries of $X$ and hence likewise for any subgroup.

We discuss the specific mechanics of the Clifford Kasparov dual to the following extent. Let $d$ be the de Rham differential on $X$, acting on $L^{2}$-forms on $X$. This Hilbert space carries a unitary action of $G$ and both $d$ and its adjoint $d^{*}$ are $G$ equivariant, and $d+d^{*}$ is an elliptic operator called the Euler (or de Rham) operator on $X$. If $\omega$ is a differential form on $X$ vanishing at infinity then the operator $\lambda_{\omega}$ of exterior product with $\omega$ defines an operator on $L^{2}$-forms which is bounded and the assignment $\omega \mapsto \lambda_{\omega}+\lambda_{\omega}^{*}$ determines a representation of $\mathscr{C}_{\tau}(X)$ which graded commutes modulo bounded operators with $d+d^{*}$. Hence we get a cycle for $\mathrm{KK}^{G}\left(\mathscr{C}_{\tau}(X), \mathbb{C}\right)$. It represents the class $D$ appearing in the Kasparov dual.

We start by recording one of the easiest computations of the Lefschetz map. The interested reader can easily prove it for his or herself using after looking briefly at the definition of the class $\Theta$ (see [13]) and reviewing the definition of the Lefschetz map. Recall that the Euler class of $X$ is the Lefschetz invariant of the identity map of $X$.

Proposition 2.4. The Euler class of $X$ is the class of the Euler operator on $X$. 
The functoriality result 1.21 combines with Proposition 2.4 to imply the homotopyinvariance of the class of the Euler operator:

$$
f_{*}\left(\operatorname{Eul}_{X}\right)=\operatorname{Eul}_{X^{\prime}}
$$

for a proper $\mathcal{G}$-equivariant homotopy-equivalence $f: X \rightarrow X^{\prime}$ between smooth and proper $\mathcal{G}$-manifolds.

We now describe the Lefschetz invariant of a more general smooth $G$-equivariant self-map of $X$. This requires a preliminary discussion.

Let $Y$ be a locally compact space and $G$ be a locally compact group acting continuously on $Y$, and let $\pi: E \rightarrow Y$ be a $G$-equivariant Euclidean $\mathbb{R}$-vector bundle over $E$. Let $A: E \rightarrow E$ be a $G$-equivariant vector bundle automorphism, that is, a continuous map $E \rightarrow E$ over $Y$ that restricts to $\mathbb{R}$-vector space isomorphisms on the fibres of $E$. We are going to define a $G$-equivariant $\mathbb{Z} / 2$-graded real line bundle $\operatorname{sign}(A)$ over $Y$. (Since we work with complex K-theory we will only use its complexification.)

If $Y$ is a point, then $G$-equivariant real vector bundles over $Y$ correspond to real orthogonal representations of $G$. The endomorphism $A$ becomes in this case an invertible linear map $A: \mathbb{R}^{n} \rightarrow \mathbb{R}^{n}$ commuting with $G$. The sign is a virtual 1-dimensional representation of $G$ and hence corresponds to a pair $(\chi, n)$, where $n \in\{0,1\}$ is the parity (we are referring to the grading, either even or odd) of the line bundle and $\chi: G \rightarrow\{-1,+1\}$ is a real-valued character. The overall parity will turn out to be 0 if $A$ preserves orientation and 1 if $A$ reverses orientation (see Example 2.6). In this sense, our invariant will refine the orientation of $A$.

As above, let $\operatorname{Cliff}(E)$ be the bundle of real Clifford algebras associated to $E$. We can also define in an analogous way $\operatorname{Cliff}(E)$ if $E$ carries an indefinite bilinear form and it is a well-known fact from algebra that if the index of the bilinear form on $E$ is divisible by 8, then the fibres of $\operatorname{Cliff}(E)$ are isomorphic to matrix algebras. In this case, a $G$-equivariant spinor bundle for $E$ is a $\mathbb{Z} / 2$-graded real vector bundle $S_{E}$ together with a grading preserving, $G$-equivariant *-algebra isomorphism $c: \operatorname{Cliff}(E) \rightarrow \operatorname{End}\left(S_{E}\right)$. This representation is determined uniquely by its restriction to $E \subseteq \operatorname{Cliff}(E)$, which is a $G$-equivariant map $c: E \rightarrow \operatorname{End}\left(S_{E}\right)$ such that $c(x)$ is odd and symmetric and satisfies $c(x)^{2}=\|x\|^{2}$ for all $x \in E$.

The spinor bundle is unique up to tensoring with a $G$-equivariant real line bundle $L$ : if $c_{t}: E \rightarrow S_{t}$ for $t=1,2$ are two $G$-equivariant spinor bundles for $E$, then we define a $G$-equivariant real line bundle $L$ over $Y$ by

$$
L:=\operatorname{Hom}_{\mathrm{Cliff}(E)}\left(S_{1}, S_{2}\right),
$$

and the evaluation isomorphism $S_{1} \otimes L \cong S_{2}$ intertwines the representations $c_{1}$ and $c_{2}$ of $\operatorname{Cliff}(E)$.

Definition 2.5. Let $A: E \rightarrow E$ be a real $G$-equivariant vector bundle automorphism and let $A=T \circ\left(A^{*} A\right)^{1 / 2}$ be its polar decomposition with an orthogonal vector bundle automorphism $T: E \rightarrow E$.

Let $F$ be another $G$-equivariant vector bundle over $Y$ with a non-degenerate bilinear form, such that the signature of $E \oplus F$ is divisible by 8 , so that $\operatorname{Cliff}(E \oplus F)$ is a bundle of matrix algebras over $\mathbb{R}$. We assume that $E \oplus F$ has a $G$-equivariant spinor bundle, that is, there exists a $G$-equivariant linear map $c: E \oplus F \rightarrow \operatorname{End}(S)$ that induces an isomorphism of graded *-algebras

$$
\operatorname{Cliff}(E \oplus F) \cong \operatorname{End}(S) .
$$

Then

$$
c^{\prime}: E \oplus F \rightarrow \operatorname{End}(S), \quad(\xi, \eta) \mapsto c(T(\xi), \eta)
$$


yields another $G$-equivariant spinor bundle for $E \oplus F$. We let

$$
\operatorname{sign}(A):=\operatorname{Hom}_{\mathrm{Cliff}(E \oplus F)}\left(\left(S, c^{\prime}\right),(S, c)\right) .
$$

This is a $G$-equivariant $\mathbb{Z} / 2$-graded real line bundle over $Y$.

It is not hard to check that $\operatorname{sign}(A)$ is well-defined and a homotopy invariant. Furthermore, $\operatorname{sign}\left(A_{1} \circ A_{2}\right) \cong \operatorname{sign}\left(A_{1}\right) \otimes \operatorname{sign}\left(A_{2}\right)$ for two equivariant automorphisms $A_{1}, A_{2}: E \rightrightarrows E$ of the same bundle, and $\operatorname{sign}\left(A_{1} \oplus A_{2}\right) \cong \operatorname{sign}\left(A_{1}\right) \otimes \operatorname{sign}\left(A_{2}\right)$ for two equivariant vector bundle automorphisms $A_{1}: E_{1} \rightarrow E_{1}$ and $A_{2}: E_{2} \rightarrow E_{2}$.

If $Y$ is a point and $\mathcal{G}$ is trivial, then $\operatorname{sign}(A)=\mathbb{R}$ for orientation-preserving $A$ and $\operatorname{sign}(A)=\mathbb{R}^{\text {op }}$ for orientation-reversing $A$, as claimed above.

Example 2.6. Consider $G=\mathbb{Z} / 2$. Let $\tau: G \rightarrow\{1\}$ be the trivial character and let $\chi: G \rightarrow\{+1,-1\}$ be the non-trivial character. Let $\mathbb{R}_{\chi}$ denote the real representation of $G$ on $\mathbb{R}$ with character $\chi$. This can be considered trivially graded; let $\mathbb{R}_{\chi}^{\text {op }}$ denote the same representation but with the opposite grading (the whole vector space is considered odd.)

Consider $A: \mathbb{R}_{\chi} \rightarrow \mathbb{R}_{\chi}, t \mapsto-t$, so $A$ commutes with $G$. Then $\operatorname{sign}(A) \cong \mathbb{R}_{\chi}^{\text {op }}$ carries a non-trivial representation.

To see this, let $F$ be $\mathbb{R}_{\chi}$ with negative definite metric. Thus the Clifford algebra of $\mathbb{R}_{\chi} \oplus \mathbb{R}_{\chi}$ is Cliff $1,1 \cong \mathbb{M}_{2 \times 2}(\mathbb{R})$. Explicitly, the map

$$
c(x, y)=\left(\begin{array}{cc}
0 & x-y \\
x+y & 0
\end{array}\right)
$$

induces the isomorphism. We equip $\mathbb{R}^{2}$ with the representation $\tau \oplus \chi$, so that $c$ is equivariant.

Twisting by $A$ yields another representation

$$
c^{\prime}(x, y):=c(-x, y)=S c(x, y) S^{-1} \quad \text { with } \quad S=S^{-1}=\left(\begin{array}{cc}
0 & 1 \\
-1 & 0
\end{array}\right) .
$$

Since $S$ reverses the grading and exchanges the representations $\tau$ and $\chi$, it induces an isomorphism $\left(\mathbb{R}_{\tau} \oplus \mathbb{R}_{\chi}^{\text {op }}\right) \otimes \mathbb{R}_{\chi}^{\text {op }} \cong \mathbb{R}_{\tau} \oplus \mathbb{R}_{\chi}^{\text {op }}$. Hence $\operatorname{sign}(A)=\mathbb{R}_{\chi}^{\text {op }}$.

Now let $X$ be a smooth Riemannian manifold and assume that $G$ acts on $X$ isometrically and continuously.

Let $\gamma: X \rightarrow X$ be a $G$-equivariant self-map of $X$. In order to write down an explicit local formula for $\operatorname{Lef}(\gamma)$, we impose the following restrictions on $\gamma$ :

- $\gamma$ is smooth;

- the fixed point subset $\operatorname{Fix}(\gamma)$ of $\gamma$ is a submanifold of $X$;

- if $(p, \xi) \in T X$ is fixed by the derivative $D \gamma$, then $\xi$ is tangent to $\operatorname{Fix}(\gamma)$.

The last two conditions are automatic if $\gamma$ is isometric with respect to some Riemannian metric (not necessarily the given one) and this of course applies by averaging the given metric if $\gamma$ has finite order.

In the simplest case, $\gamma$ and $\mathrm{id}_{X}$ are transverse, that is, id $-D \gamma$ is invertible at each fixed point of $\gamma$; this implies that $\gamma$ has isolated fixed points.

To describe the Lefschetz invariant, we abbreviate $Y:=\operatorname{Fix}(\gamma)$. This is a closed submanifold of $X$ by assumption. Let $\nu$ be the normal bundle of $Y$ in $X$. Since the tangent space of $Y$ is left fixed by the derivative $D \gamma$, it induces a linear map $D_{\nu} \gamma: \nu \rightarrow \nu$. By assumption, the map $\operatorname{id}_{\nu}-D_{\nu} \gamma: \nu \rightarrow \nu$ is invertible.

Theorem 2.7. Let $X$ be a complete smooth Riemannian manifold, let $G$ be a locally compact group that acts on $X$ smoothly and by isometries, and let $\gamma: X \rightarrow X$ be a self-map satisfying the three conditions enumerated above. Let $\nu$ be the normal bundle of $Y$ in $X$ and let $D_{\nu} \gamma: \nu \rightarrow \nu$ be induced by the derivative of $\gamma$ as above. 
Let $r_{Y}: \mathcal{C}_{0}(X) \rightarrow \mathcal{C}_{0}(Y)$ be the restriction map and let $\operatorname{Eul}_{Y} \in \operatorname{KK}_{0}^{G}\left(\mathcal{C}_{0}(Y), \mathbb{1}\right)$ be the equivariant Euler characteristic of $Y$. Then

$$
\operatorname{Lef}(\gamma)=r_{Y} \otimes_{\mathcal{C}_{0}(Y)} \operatorname{sign}\left(\operatorname{id}_{\nu}-D_{\nu} \gamma\right) \otimes_{\mathcal{C}_{0}(Y)} \operatorname{Eul}_{Y}
$$

Furthermore, Eul $_{Y}$ is the equivariant K-homology class of the de Rham operator on $Y$.

In brief, the Lefschetz invariant of $\gamma$ is the Euler characteristic of the fixed-point set, twisted by an appropriate equivariant line bundle depending on orientation data.

If $\gamma$ and $\operatorname{id}_{X}$ are transverse then the fixed point subset $Y$ is discrete. A discrete set is a manifold and its Euler characteristic - a degenerate case of Proposition 2.4 - is represented by the Kasparov cycle in which the Hilbert space is $L^{2}\left(\Lambda_{\mathbb{C}}^{*}\left(T^{*} Y\right)\right):=\ell^{2}(Y)$ equipped with the representation $\mathcal{C}_{0}(Y) \rightarrow \mathbb{K}\left(\ell^{2} Y\right)$ by pointwise multiplication operators, and the zero operator. The group permutes the points of $Y$ and so acts by unitaries on $\ell^{2}(Y)$.

The normal bundle $\nu$ to $Y$ in $X$ in this case is the restriction of the vector bundle $\mathrm{T} X$ to the subset $Y$. For $p \in Y$, let $n_{p}$ be +1 if $\operatorname{id}_{T_{p} X}-D_{p} \gamma$ preserves orientation, and -1 otherwise. The graded equivariant line bundle $\operatorname{sign}\left(\operatorname{id}_{\nu}-D_{\nu} \gamma\right)$ in Theorem 2.7 is determined by pairs $\left(n_{p}, \chi_{p}\right)$ for $p \in Y$, where $n_{p}$ is the parity of the representation at $p$ and $\chi_{p}$ is a certain real-valued character $\chi_{p}: \operatorname{Stab}(p) \rightarrow$ $\{-1,+1\}$ that depends on $\operatorname{id}_{\mathrm{T}_{p} X}-D_{p} \gamma$ and the representation of the stabiliser $\operatorname{Stab}(p) \subseteq G$ on $\mathrm{T}_{p} X$. Equivariance implies that $n_{p}$ is constant along $G$-orbits, whereas $\chi_{p}$ behaves like $\chi_{g \cdot p}=\chi_{p} \circ \operatorname{Ad}\left(g^{-1}\right)$. Let $\ell_{\chi}^{2}(G p)$ be the representation of the cross-product $G \ltimes \mathcal{C}_{0}(G / \operatorname{Stab}(p))$ obtained by inducing the representation $\chi_{p}$ from $\operatorname{Stab}(p)$, and let $\mathcal{C}_{0}(X)$ act on $\ell_{\chi}^{2}(G p)$ by restriction to $G / \operatorname{Stab}(p)$. This defines a $G$-equivariant *-homomorphism

$$
\xi_{G p, \chi}: \mathcal{C}_{0}(X) \rightarrow \mathbb{K}\left(\ell_{\chi}^{2} G\right) .
$$

Theorem 2.7 asserts the following:

Corollary 2.8. If the graph of $\phi$ is transverse to the diagonal in $X \times X$ then,

$$
\operatorname{Lef}(\gamma)=\sum_{G p \in G \backslash \operatorname{Fix}(\gamma)} n_{p}\left[\xi_{G p, \chi}\right]
$$

where $\left[\xi_{G p, \chi}\right] \in \operatorname{KK}_{0}^{G}\left(\mathcal{C}_{0}(X), \mathbb{1}\right)$ and the multiplicities $n_{p}$ are explained above.

Furthermore, the character $\chi: \operatorname{Stab}_{G}(p) \rightarrow\{-1,+1\}$ at a fixed point $p$ has the explicit formula

$$
\chi(g)=\operatorname{sign} \operatorname{det}\left(\mathrm{id}-D_{p} \phi\right) \cdot \operatorname{sign} \operatorname{det}\left(\mathrm{id}-\left.D_{p} \phi\right|_{\operatorname{Fix}(g)}\right) .
$$

If, in addition, $G$ is trivial and $X$ is connected, then $\xi_{G p, \chi}=\operatorname{ev}_{p}$ for all $p \in Y$; moreover, all point evaluations have the same K-homology class because they are homotopic. Hence we get the classical Lefschetz data multiplied by the K-homology class of a point

$$
\operatorname{Lef}(\gamma)=\left(\sum_{p \in \operatorname{Fix}(\gamma)} \operatorname{sign}\left(\operatorname{id}_{T_{p} X}-D \gamma_{p}\right)\right) \cdot[\mathrm{ev}]
$$

as asserted above. This sum is finite if $X$ is compact.

We include the following for the benefit of the reader; it enables her or she to verify our computations by direct inspection.

Lemma 2.9. Let $H \subseteq G$ be compact and open, let $p, q \in X^{H}$ belong to the same path component of the fixed point subspace $X^{H}$, and let $\chi \in \operatorname{Rep}(H)$. Then

$$
\left[\xi_{G p, \operatorname{Ind}_{H}^{\operatorname{Stab}(G p)}(\chi)}\right]=\left[\xi_{G q, \operatorname{Ind}_{H}^{\operatorname{Stab}(G q)}(\chi)}\right] \quad \text { in } \mathrm{KK}_{0}\left(\mathcal{C}_{0}(X), \mathbb{1}\right) .
$$


Remark 2.10. If the identity map id: $X \rightarrow X$ can be equivariantly perturbed to be in general position in the sense explained above, then combining Proposition 2.4 and Corollary 2.8 proves that the class of the de Rham operator in $\mathrm{KK}^{G}\left(\mathcal{C}_{0}(X), \mathbb{C}\right)$ is a sum of point-evaluation classes. Lück and Rosenberg show in [23] that this can always be achieved when $G$ is discrete and acts properly on $X$.

The next two explicit examples involve isolated fixed points.

Example 2.11. Let $G \cong \mathbb{Z} \ltimes \mathbb{Z} / 2 \mathbb{Z}$ be the infinite dihedral group, identified with the group of affine transformations of $\mathbb{R}$ generated by $u(x)=-x$ and $w(x)=x+1$. Then $G$ has exactly two conjugacy classes of finite subgroups, each isomorphic to $\mathbb{Z} / 2$. Its action on $\mathbb{R}$ is proper, and the closed interval $[0,1 / 2]$ is a fundamental domain. There are two orbits of fixed point in $\mathbb{R}$, those of 0 and $1 / 2$, and their stabilisers represent the two conjugacy classes of finite subgroups.

Now we use some notation from Example 2.6. Each copy of $\mathbb{Z} / 2$ acting on the tangent space at the fixed point acts by multiplication by -1 on tangent vectors. Therefore, the computations in Example 2.6 show that for any nonzero real number $A$, viewed as a linear transformation of the tangent space that commutes with $\mathbb{Z} / 2$, we have

$$
\operatorname{sign}(A)= \begin{cases}\mathbb{R}_{\chi}^{\text {op }} & \text { if } A<0, \text { and } \\ \mathbb{R}_{\tau} & \text { if } A>0 .\end{cases}
$$

Let $\phi$ be a small $G$-equivariant perturbation of the identity map $\mathbb{R} \rightarrow \mathbb{R}$ with the following properties. First, $\phi$ maps the interval $[0,1 / 2]$ to itself. Secondly, its fixed points in $[0,1 / 2]$ are the end points $0,1 / 2$, and $1 / 4$; thirdly, its derivative is bigger than 1 at both endpoints and between 0 and 1 at $1 / 4$. Such a map $\phi$ clearly exists. Furthermore, it is homotopic to the identity map, so that $\operatorname{Lef}(\phi)=\operatorname{Eul}_{\mathbb{R}}$.

By construction, there are three fixed points modulo $G$, namely, the orbits of $0,1 / 4$ and $1 / 2$. The isotropy groups of the first and third orbit are non-conjugate subgroups isomorphic to $\mathbb{Z} / 2$; from Example 2.6 each of them contributes $\mathbb{R}_{\chi}^{\text {op }}$. The point $1 / 4$ contributes the trivial character of the trivial subgroup. Hence

$$
\operatorname{Lef}(\phi)=-\left[\xi_{\mathbb{Z}, \chi}\right]-\left[\xi_{\mathbb{Z}+1 / 2, \chi}\right]+\left[\xi_{\mathbb{Z}+1 / 4}\right] .
$$

On the other hand, suppose we change the above map $\phi$ to fix the same points but to have zero derivative at 0 and $1 / 2$ and large derivative at $1 / 4$. This is obviously possible. Then we get contributions of $\mathbb{R}_{\tau}$ at 0 and $1 / 2$ and a contribution of $-\left[\xi_{1 / 4}\right]$ at $1 / 4$. Hence

$$
\operatorname{Lef}(\phi)=\left[\xi_{\mathbb{Z}, \tau}\right]+\left[\xi_{\mathbb{Z}+1 / 2, \tau}\right]-\left[\xi_{\mathbb{Z}+1 / 4}\right]
$$

Combining both formulas yields the identity

$$
\left[\xi_{\mathbb{Z}, \tau}\right]+\left[\xi_{\mathbb{Z}+1 / 2, \tau}\right]-\left[\xi_{\mathbb{Z}+1 / 4}\right]=-\left[\xi_{\mathbb{Z}, \chi}\right]-\left[\xi_{\mathbb{Z}+1 / 2, \chi}\right]+\left[\xi_{\mathbb{Z}+1 / 4}\right]
$$

By the way, the left-hand side is the description of $\operatorname{Eul}_{\mathbb{R}}$ we get from the combinatorial dual with the obvious $G$-invariant triangulation of $\mathbb{R}$ with vertex set $\mathbb{Z}+1 / 2 \subset \mathbb{R}$.

Using Lemma 2.9 one can check (2.12) by direct computation.

\section{Geometric KK-Theory}

In the previous chapter we explained our computation of the Lefschetz map for self-maps of a $\mathcal{G}$-space $X$ in several relatively simple situations. Of course, not all equivariant Kasparov self-morphisms $\mathrm{KK}_{*}^{\mathcal{G}}\left(\mathcal{C}_{0}(X), \mathcal{C}_{0}(X)\right)$ are represented by maps. We have organized this survey around the problem of computing Lefschetz invariants of more general equivariant Kasparov self-morphisms. This requires describing the morphisms themselves in some geometric way. In order to do this, we have extended the theory of correspondences developed by Baum, Connes and Skandalis (see [3] and 8]) to work equivariantly. This extension is somewhat involved, and we 
will devote some space to explaining it. With an appropriate equivalence relation on equivariant correspondences, they make up a category with the same formal properties as $\mathrm{KK}^{\mathcal{G}}$ and which maps to it, in some cases isomorphically, and this will allow us to completely compute the Lefschetz map in geometric, or topological terms.

In the previous sections, we allowed arbitrary groupoids $\mathcal{G}$ but required actions to be essentially proper. It turns out that the mechanics of the topological theory requires an even stronger assumption: that the groupoid $\mathcal{G}$ is itself be a proper groupoid. This is of course very restrictive: if $\mathcal{G}$ is also a group, then it must be compact, for example. However, for some purposes, restriction to proper groupoids makes no difference. If $G$ is an arbitrary groupoid and if $\mathcal{E} G$ is its classifying space for proper actions, then the transformation groupoid $\mathcal{G}:=G \ltimes \mathcal{E} G$ is a proper groupoid. If we are interested in a specific action of $G$ on $X$, then to get into our framework, we thus replace $G$ by $\mathcal{G}$ and replace a given $G$-space $X$ with the $\mathcal{G}$-space $X \times \mathcal{E} G$. The precise extent to which this involves no loss of information is governed by the apparatus of the Baum-Connes conjecture; we omit details here. In any case, this reduction trick forces us to work in the context of groupoids even if we are initially only interested in groups.

3.1. Equivariant vector bundles. As above, $\mathcal{G}$ is a locally compact Hausdorff proper groupoid with Haar system. If $X$ is a $\mathcal{G}$-space, we remind the reader that the anchor map for the action is denoted $\varrho_{X}: X \rightarrow Z$. A $\mathcal{G}$-equivariant vector bundle on $X$ is a vector bundle on $X$ which is also a $\mathcal{G}$-space such that elements of $\mathcal{G}$ map fibres to fibres linearly. Thus, if $\mathcal{G}$ is a compact group, then a $\mathcal{G}$-equivariant vector bundle on a point is a finite-dimensional linear representation of $\mathcal{G}$. If $G$ is a locally compact group and $\mathcal{E} G$ is its classifying space, $\mathcal{G}:=G \ltimes \mathcal{E} G$, so that $Z=\mathcal{E} G$, then a $\mathcal{G}$-equivariant vector bundle on $Z$ is the same as a $G$-equivariant vector bundle on $Z$.

Given that $\mathcal{G}$ is assumed proper, equivariant vector bundles behave in some ways just like ordinary vector bundles. For example, if $Y \subset X$ is a closed, $\mathcal{G}$-invariant subset of a $\mathcal{G}$-space $X$, and if $V$ is a $\mathcal{G}$-equivariant vector bundle on $Y$, then any equivariant section of $V$ defined on $Y$ can be extended to an equivariant section defined on an open $\mathcal{G}$-invariant neighbourhood of $Y$. This involves an averaging procedure (see [16]). As a consequence, if $f_{i}: X \rightarrow Y, i=0,1$ are two $\mathcal{G}$-equivariantly homotopic maps, and if $V$ is a $\mathcal{G}$-equivariant vector bundle on $Y$, then $f_{0}^{*}(V)$ and $f_{1}^{*}(V)$ are $\mathcal{G}$-equivariantly isomorphic.

On the other hand, some new problems appear in connection with equivariant vector bundles.

Definition 3.1. Let $X$ be a $\mathcal{G}$-space. A $\mathcal{G}$-vector bundle over $X$ is trivial if it is pulled back from $Z$ under the anchor map $\varrho_{X}: X \rightarrow Z$. We denote the pull-back of a $\mathcal{G}$-vector bundle $E$ over $Z$ to the $\mathcal{G}$-space $X$ by $E^{X}$. A $\mathcal{G}$-equivariant vector bundle is subtrivial if it is a direct summand of a trivial $\mathcal{G}$-vector bundle.

For example, if $\mathcal{G}$ is a compact group, then a trivial $\mathcal{G}$-equivariant vector bundle over $X$ has the form $X \times \mathbb{R}^{n}$ where $\mathbb{R}^{n}$ carries a linear representation of $\mathcal{G}$, and where $\mathcal{G}$ acts on $X \times \mathbb{R}^{n}$ diagonally.

We will make repeated use of the following basic fact about representations of compact groups.

Lemma 3.2. Let $\mathcal{G}$ be a compact group and $\mathcal{G}^{\prime} \subset \mathcal{G}$ be a subgroup. Then any finite-dimensional representation of $\mathcal{G}^{\prime}$ is contained in the restriction to $\mathcal{G}^{\prime}$ of a finite-dimensional representation of $\mathcal{G}$.

Due to the notion of 'trivial' vector bundle we are using, not every $\mathcal{G}$-vector bundle is locally trivial in the category of $\mathcal{G}$-vector bundles, i.e. locally isomorphic 
to a trivial $\mathcal{G}$-vector bundle. But it is not hard to prove the following and it is a good exercise for understanding equivariant vector bundles. We leave the proof to the reader, but see $\$ 3.2$ for some important ingredients in the argument.

Lemma 3.3. Every $\mathcal{G}$-equivariant vector bundle on $X$ is locally subtrivial in the sense that for every $x \in X$ there is a $\mathcal{G}$-equivariant vector bundle on $Z$, a $\mathcal{G}$ equivariant neighbourhood $U$ of $x$, and an embedding $\varphi: V_{\left.\right|_{U}} \rightarrow E^{U}$.

Improving this local result to a global one is not possible, however, without an appropriate compactness assumption.

Example 3.4. Let $X:=\mathbb{Z}$ with the trivial action of the compact group $\mathcal{G}:=\mathbb{T}$. Then the 1-dimensional complex vector bundle $\mathbb{Z} \times \mathbb{C}$ with the action of $z \in \mathcal{G}$ in the fibre over $n$ by the character $z \mapsto z^{n}$ is not subtrivial, since it contains infinitely many distinct irreducible representations of $\mathcal{G}$.

Definition 3.5. Let $X$ be a $\mathcal{G}$-space.

- The space $X$ has enough $\mathcal{G}$-equivariant vector bundles if whenever we are given $x \in X$ and a finite-dimensional representation of the compact isotropy group $\mathcal{G}_{x}^{x}$ of $x$, there is a $\mathcal{G}$-equivariant vector bundle over $X$ whose restriction to $x$ contains the given representation of $\mathcal{G}_{x}^{x}$.

- The space $X$ has a full vector bundle if there is a vector bundle $V$ over $X$ such that any irreducible representation of $\mathcal{G}_{x}^{x}$ is contained in the representation of $\mathcal{G}_{x}^{x}$ on $V_{x}$ (and we say that such a vector bundle $V$ is full.)

It is the content of Lemma 3.2 that a compact group acting on a point has enough vector bundles.

It is easy to check that if $f: X \rightarrow Y$ is a $\mathcal{G}$-equivariant map then $X$ has enough equivariant vector bundles if $Y$ does, and $f^{*}(V)$ is a full vector bundle on $X$ if $V$ is a full vector bundle on $Y$. Both of these assertions use the basic fact Lemma 3.2

Example 3.6. The $\mathcal{G}$-space described in Example 3.4 does not have a full vector bundle, although by Lemma 3.2 it does have enough vector bundles.

The following example is more subtle. It is due to Julianne Sauer (see [30]).

Example 3.7. Let $X=\mathbb{R}$ and $K$ be the compact group $K:=\prod_{n \in \mathbb{Z}} \mathbb{Z} / 2$ acting trivially on $\mathbb{R}$. Since $X$ is $K$-equivariantly contractible, and by the homotopyinvariance of equivariant vector bundles (mentioned above at the beginning of \$3.1), any $K$-equivariant vector bundle $V$ on $X$ is trivial, and hence all the representations of $K$ on the fibres $V_{x}$ are equivalent.

Now let $\sigma: K \rightarrow K$ be the shift (left) automorphism and consider the group $G:=K \ltimes_{\sigma} \mathbb{Z}$; it acts on $X$ by letting $\sigma(t):=t+1$. This is a proper action of a locally compact group on $X$ by homeomorphisms. Of course as a groupoid $G$ is not proper, but we repair this below.

We claim that the only trivial $G$-vector bundles on $X$ yield trivial $K$-representations in their fibres. This will show that $X$ does not have enough $G$-vector bundles.

The proof is as follows: any $G$-vector bundle $V$ on $X$ is trivial as a $K$-vector bundle, by the above results. Hence all the representations of $K$ one gets by considering fibres are equivalent. On the other hand, the covariance rule for the semi-direct product implies that representations at $x$ and $x+1$ are mapped to each other (up to equivalence) by the action of $\sigma$, and therefore $\hat{\sigma}: \operatorname{Rep}(K) \rightarrow \operatorname{Rep}(K)$ has a fixedpoint. But $\operatorname{Rep}(K)$ is the direct sum of $\widehat{\mathbb{Z} / 2}$ 's and $\widehat{\sigma}$ acts as the shift. The only fixed point then is the zero sequence. This corresponds to a trivial representation. This means that at every point $x \in X$ the representation of $K$ we get on $V_{x}$ is trivial. 
To repair the non-properness of $G$, replace it by $\mathcal{G}:=G \ltimes \mathcal{E} G$ and replace $X$ by $X \times \mathcal{E} G$ as explained at the beginning of this section, then we get an example of a proper groupoid $\mathcal{G}$ and a $\mathcal{G}$-space $X \times \mathcal{E} G$ such which does not have enough equivariant vector bundles. This is because $X \times \mathcal{E} G$ and $X$ are $G$-equivariantly homotopy-equivalent anyway, because the action of $G$ on $X$ is proper. Hence these spaces have the same categories of equivariant vector bundles.

A Morita-equivalent approach is via a mapping cyclinder construction. Take $[0,1] \times K$ modulo the relation $(1, k) \sim(0, \sigma(k))$. This results in a bundle of compact groups over the circle which can shown to be locally compact groupoid with Haar system.

Let $\mathcal{G}$ be this groupoid: it is proper. Its base $Z$ is the circle. By a holonomy argument similar to the one just given, any $\mathcal{G}$-equivariant vector bundle over $Z$ must restrict in each fibre to a trivial representation of $K$. Thus, there are not enough $\mathcal{G}$-equivariant vector bundles on $Z$.

Example 3.8. If $\Gamma$ is a discrete group with a $\Gamma$-compact model for $\mathcal{E} \Gamma$, then Lück and Oliver have shown in 22] that there is a full $\mathcal{G}$-equivariant vector bundle on $Z$, where $\mathcal{G}:=\Gamma \ltimes \mathcal{E} \Gamma$, (so that $Z=\mathcal{E} \Gamma$.)

3.2. Equivariant embedding theorems and topological index. We now indicate why the condition of having enough vector bundles, or having a full vector bundle, is important for describing analytic equivariant KK-groups topologically.

We start with the problem, famously treated by Atiyah and Singer, of describing the equivariant (analytic) index of a $\mathcal{G}$-equivariant elliptic operator in topological terms, where $\mathcal{G}$ is a compact group, keeping in mind that an equivariant elliptic operator is a prototypical cycle for equivariant KK-theory.

Let $X$ be a smooth manifold with a smooth action of the compact group $\mathcal{G}$. The symbol of an equivariant elliptic operator on $X$ is an equivariant $\mathrm{K}$-theory class for $\mathrm{T} X$. The idea of Atiyah and Singer for defining the topological index of the operator is to smoothly embed $X$ in a finite-dimensional (linear) representation of $\mathcal{G}$ on $\mathbb{R}^{n}$. The derivative of this embedding gives a smooth embedding of $\mathrm{T} X$ in $\mathbb{R}^{2 n}$, where $\mathrm{T} X$ has the induced action of $\mathcal{G}$. Since $\mathrm{T} X$ is an equivariantly K-oriented manifold, the normal bundle $\nu$ to the embedding is a $\mathcal{G}$-equivariantly $\mathrm{K}$-oriented vector bundle on $\mathrm{T} X$. The tubular neighbourhood embedding identities it with an open, $\mathcal{G}$-equivariant neighbourhood of the image of $\mathrm{T} X$ in $\mathbb{R}^{2 n}$. We now obtain a composition

$$
\mathrm{K}_{\mathcal{G}}^{*}(\mathrm{~T} X) \rightarrow \mathrm{K}_{\mathcal{G}}^{*}(N) \rightarrow \mathrm{K}_{\mathcal{G}}^{*}\left(\mathbb{R}^{2 n}\right) \rightarrow \mathrm{K}_{\mathcal{G}}^{*}(\star) \cong \operatorname{Rep}(\mathcal{G}),
$$

where the first map is the Thom isomorphism for the equivariantly K-oriented $\mathcal{G}$ vector bundle $N$, the second is the map on equivariant K-theory induced by the open inclusion $N \hookrightarrow \mathbb{R}^{2 n}$ and the third is equivariant Bott Periodicity ( $\mathbb{R}^{2 n}$ with the diagonal action of $\mathcal{G}$ has an equivariant complex structure, so a $\mathcal{G}$-equivariant spin $^{c}$-structure).

The content of the index theorem is that this composition agrees with the map $\mathrm{K}_{\mathcal{G}}^{*}(\mathrm{~T} X) \rightarrow \operatorname{Rep}(\mathcal{G})$ obtained by first interpreting cycles for $\mathrm{K}_{\mathcal{G}}^{*}(\mathrm{~T} X)$ as symbols of equivariant elliptic operators on $X$, making these elliptic operators into Fredholm operators, and taking their equivariant indices.

But how do we get a smooth, equivariant embedding of $X$ in a finite-dimensional linear representation of $\mathcal{G}$ in the first place? Since it involves important ideas for us, we will sketch the proof. Very similar arguments also prove Lemma 3.3.

First of all, we may assume (or average using the Haar system on $\mathcal{G}$ ) that $X$ has an invariant Riemannian metric. Now the orbit $\mathcal{G} x$ is a smooth embedded submanifold of $X$ isomorphic to $\mathcal{G} / \mathcal{G}_{x}^{x}$. The tangent space of $X$ at $x$ splits into 
the orthogonal sum of the tangent space to the orbit and its orthogonal complement $N_{x}:=T_{x}(\mathcal{G} x)^{\perp}$. The latter is a finite-dimensional representation of $\mathcal{G}_{x}^{x}$, and inducing it results in a $\mathcal{G}$-equivariant vector bundle

$$
N:=\mathcal{G} \times \mathcal{G}_{x}^{x} N_{x}:=\mathcal{G} \times N_{x} /(g, n) \sim\left(g h, h^{-1} n\right) \text { for } h \in \mathcal{G}_{x}^{x}
$$

on the orbit which is precisely the normal bundle to the embedded submanifold $\mathcal{G} x$. By exponentiating we obtain an equivariant diffeomorphism between the total space of $N$ and an invariant open neighbourhood $U$ of the orbit. We embed this neighbourhood as follows.

By Lemma 3.2 the representation of $\mathcal{G}_{x}^{x}$ on $N_{x}$ is contained in the restriction of some representation of $\mathcal{G}$ on some finite-dimensional vector space $\tilde{N}_{x}$. The naturality of induction implies that we have an inclusion of vector bundles $N \subset \tilde{N}:=$ $\mathcal{G} \ltimes_{\mathcal{G}_{x}^{x}} \tilde{N}_{x}$. But since $\tilde{N}_{x}$ is the restriction of a $\mathcal{G}$-representation, $\tilde{N}$ is a product bundle, i.e. a trivial $\mathcal{G}$ vector bundle on the orbit. This provides a $\mathcal{G}$-equivariant map $U \rightarrow \tilde{N}_{x}$, explicitly, by mapping $[(g, n)] \in U \cong N \subset \tilde{N}$ to the point $g n \in \tilde{N}_{x}$. It is of course not necessarily an embedding; to improve it to an embedding, fix a vector $v \in \tilde{N}_{x}$ whose isotropy in $\mathcal{G}$ is exactly $\mathcal{G}_{x}^{x}$ (for this see also [27]) and set

$$
\varphi: U \cong \mathcal{G} \times_{\mathcal{G}_{x}^{x}} N_{x} \rightarrow \tilde{N}_{x} \oplus \tilde{N}_{x}, \quad \varphi([g, n]):=(g n, g v) .
$$

The map $\varphi$ is an equivariant embedding as required.

As mentioned, if $X$ is compact, then we can then we can (carefully) paste together the local embeddings to get an embedding of $X$; see [16], or the source of this theorem, 27. The reader should notice that the assumption that $X$ has enough vector bundles is used implicitly to show that the representation of $\mathcal{G}_{x}^{x}$ on $N_{x}$ can be extended to a $\mathcal{G}$-equivariant vector bundle on the orbit of $x$ (the vector bundle $\tilde{N}$ induced from $\tilde{N}_{x}$ ). This was the statement of Lemma 3.2, and is just an explicit way of saying that $\mathcal{G}$ has enough vector bundles on its one-point base space.

More generally, in [16] the following is proved. Let $X$ be a $\mathcal{G}$-space, where $\mathcal{G}$ is a proper groupoid.

We say that $X$ is a smooth $\mathcal{G}$-manifold if we can cover $X$ by charts of the form $U \times \mathbb{R}^{n}$ where $U \subset Z$ is open, so that with respect to this product structure the anchor map $\varrho_{X}: X \rightarrow Z$ identifies with the first coordinate projection, and such that groupoid elements and change of coordinates are smooth in the vertical direction.

Theorem 3.9. Let $\mathcal{G}$ be a (proper) groupoid and $X$ and $Y$ be smooth $\mathcal{G}$-manifolds. Suppose that either

A. The $\mathcal{G}$-space $Z$ has enough vector bundles and $\mathcal{G} \backslash X$ is compact,

B. $Z$ has a full vector bundle and $\mathcal{G} \backslash X$ has finite covering dimension.

Then, given a smooth, $\mathcal{G}$-equivariant map $f: X \rightarrow Y$, there exists

- A smooth $\mathcal{G}$-equivariant vector bundle $V$ over $X$,

- A smooth $\mathcal{G}$-equivariant vector bundle $E$ over $Z$,

- An smooth, equivariant open embedding $\varphi: V \rightarrow E^{Y}$, such that

$$
f=\pi_{E^{Y}} \circ \varphi \circ \zeta_{V}
$$

Furthermore, under the union of hypotheses $\mathbf{A}, \mathbf{B}$, any $\mathcal{G}$-equivariant vector bundle over $X$ (or $Y)$ is subtrivial.

Recall that the notation $E^{Y}$ means the pullback of $E$ to $Y$ using the anchor map $\varrho_{Y}: Y \rightarrow Z$.

We call a factorisation of a map $f: X \rightarrow Y$ of the form 3.10 a normal factorisation. 
3.3. Normal maps. As in the previous section, $\mathcal{G}$ is a proper groupoid. The constructions of the previous section motivate the following definition.

Definition 3.11. A $\mathcal{G}$-equivariant normal map $\Phi$ from $X$ to $Y$ is a triple $(V, E, \hat{f})$ where $V$ is a $\mathcal{G}$-equivariant subtrivial vector bundle over $X, E$ is a $\mathcal{G}$-equivariant vector bundle over $Z$ and $\hat{f}: V \rightarrow E^{Y}$ is a $\mathcal{G}$-equivariant open embedding.

- The trace of $\Phi$ is the composition $\pi_{E^{Y}} \circ \hat{f} \circ \zeta_{V}$.

- The stable normal bundle of $\Phi$ is the class $[V]-\left[E^{X}\right] \in \mathrm{VK}_{\mathcal{G}}(X)$ (the Grothendieck group of isomorphism classes of $\mathcal{G}$-equivariant real vector bundles over $X$.)

- The degree of $\Phi$ is $\operatorname{dim}(V)-\operatorname{dim}(E)$.

- The normal map $\Phi$ is $\mathrm{K}$-oriented if the $V$ and $E$ are equivariantly Koriented.

- The normal map $\Phi$ is smooth if $X$ and $Y$ are smooth $\mathcal{G}$-manifolds and $V$ and $E$ are smooth equivariant vector bundles on which $\mathcal{G}$ acts smoothly, and if $\hat{f}$ is a smooth map.

Of course the trace of a $\mathcal{G}$-equivariant smooth normal map is itself a smooth equivariant map, and the content of Theorem 3.9 is that, conversely, any smooth equivariant map between smooth $\mathcal{G}$-manifolds is the trace of some smooth normal map, under some hypotheses about the availability of equivariant vector bundles.

Example 3.12. The simplest example of a normal map is the zero section and bundle projection

$$
\zeta_{V}: X \rightarrow V, \quad \pi_{V}:|V| \rightarrow X,
$$

of a $\mathcal{G}$-equivariant vector bundle $V$ over a compact $\mathcal{G}$-space $X$, where $\mathcal{G}$ is a compact group. The zero section is the trace of the normal map $\left(V, \mathrm{id}, 0_{|V|}\right)$. The stable normal bundle is the class $[V]$ of the vector bundle itself. Since $X$ is compact, $V$ is subtrivial. If $X$ is not compact, this can fail, c.f. Example 3.4 what is required for that is a full vector bundle on $Z$.

With the same (compact) $X, V$ etc, the bundle projection $\pi_{V}:|V| \rightarrow X$ is the trace of a normal map $\left(\pi_{V}^{*}\left(V^{\prime}\right)\right.$, id, $\left.\mathbb{R}^{n}\right)$, where $V^{\prime} \in \operatorname{Vect}_{\mathcal{G}}(X)$ is a choice of $\mathcal{G}$-vector bundle on $X$ such that $V \oplus V^{\prime}$ is a trivial bundle $\left(\mathbb{R}^{n}\right)^{X}$. Here we identify implicitly the total spaces of the vector bundle $\pi_{V}^{*}\left(V^{\prime}\right)$ over $|V|$ and the trivial vector bundle $V \oplus V^{\prime} \cong \mathbb{R}^{n}$ over $X$. The stable normal bundle is thus $\pi_{V}^{*}\left(\left[V^{\prime}\right]\right)-\left[\left(\mathbb{R}^{n}\right)^{X}\right] \in$ $\mathrm{VK}_{\mathcal{G}}(|V|)$ respectively.

Example 3.13. Let $\mathcal{G}$ be a compact group acting smoothly on manifolds $X, Y$ with $X$ compact. By the discussion in $\$ 3.2$ we can fix a smooth, equivariant embedding $i: X \rightarrow E$ in a linear representation of $\mathcal{G}$. Define $V$ to be the normal bundle to the embedding $x \mapsto(f(x), i(x))$ of $X$ in $E^{Y}:=Y \times E$. Let $\varphi: V \rightarrow E^{Y}$ be the corresponding tubular neighbourhood embedding. Then the trace of the composition $\pi_{E^{Y}} \circ \varphi \circ \zeta_{V}$ of the normal map $(V, E, \hat{f})$ is $f$. Since $\mathrm{T} X \oplus V \cong$ $f^{*}(\mathrm{~T} Y) \oplus E^{X}$, the stable normal bundle is $f^{*}([\mathrm{~T} Y])-[\mathrm{T} X] \in \mathrm{VK}_{\mathcal{G}}(X)$.

Example 3.14. If $\Gamma$ is a discrete group with a $\Gamma$-compact model for $\mathcal{E} \Gamma$, then Lück and Oliver have shown in 22] that there is a full $\mathcal{G}$-equivariant vector bundle on $Z$, where $\mathcal{G}:=\Gamma \ltimes \mathcal{E} \Gamma$, (so that $Z=\mathcal{E} \Gamma$.) Let $X$ and $Y$ be smooth manifolds equipped with smooth actions of $\Gamma$. As above let $\mathcal{G}$ be the proper groupoid $\Gamma \times \mathcal{E} \Gamma$. The $\mathcal{G}$ spaces $X \times \mathcal{E} \Gamma$ and $Y \times \mathcal{E} \Gamma$ are smooth $\mathcal{G}$-manifolds and $f \times \mathrm{id}_{\mathcal{E} \Gamma}: X \times \mathcal{E} \Gamma \rightarrow Y \times \mathcal{E} \Gamma$ is a smooth $\mathcal{G}$-equivariant map which is the trace of a normal map because of Theorem 3.9 and the result of Lück and Oliver.

As we will see in the next section, if $f$ is also K-orientable in an appropriate sense, then it will give rise to a morphism in equivariant $\mathrm{KK}^{\Gamma}$, using the normal factorisation. 
Two normal maps are isomorphic if there are vector bundle isomorphisms $V_{0} \cong V_{1}$ and $E_{0} \cong E_{1}$ that intertwine the open embeddings $f_{0}$ and $f_{1}$. The lifting of a normal map $\Phi=\Psi=(V, \varphi, E)$ along an equivariant vector bundle $E^{+}$over $Z$ is the normal $\operatorname{map} \Phi \oplus E^{+}:=\left(V \oplus\left(E^{+}\right)^{X}, E \oplus E^{+}, \hat{f} \times_{Z} \mathrm{id}_{E^{+}}\right)$. Two normal maps are stably isomorphic if there are $\mathcal{G}$-equivariant vector bundles $E_{0}^{+}$and $E_{1}^{+}$such that $\Phi_{0} \oplus E_{0}^{+}$ is isomorphic to $\Phi_{1} \oplus E_{1}^{+}$. Finally, two normal maps $\Phi_{0}$ and $\Phi_{1}$ are isotopic if there is a continuous 1-parameter family of normal maps whose values at the endpoints are stably isomorphic to $\Phi_{0}$ and $\Phi_{1}$ respectively (see [16] for the exact definition), and are equivalent if they have isotopic liftings.

A normal map $(V, E, \hat{f})$ between smooth $\mathcal{G}$-manifolds is smooth if $V$ is a smooth vector bundle and $\hat{f}$ is a (fibrewise) diffeomorphism onto its range. There is an obvious notion of smooth equivalence of normal maps.

3.4. Manifolds with smooth normal maps to $Z$. A useful hypothesis covering a number of geometric situations is that a given smooth $\mathcal{G}$-manifold $X$ admits a smooth normal map to the object space $Z$ of $\mathcal{G}$. By the theorem above this is the case if $\mathbf{A}$ or $\mathbf{B}$ hold. It means explicitly that we have a triple $\left(\mathrm{N}_{X}, \hat{g}, E\right)$ where $\mathrm{N}_{X}$ is a smooth subtrivial vector bundle over $X, E$ is an equivariant vector bundle over $Z$ and $\hat{g}$ is a smooth open equivariant embedding $\mathrm{N}_{X} \rightarrow E$. Note that $\mathrm{N}_{X} \oplus \mathrm{T} X \cong E^{X}$. Such a normal map is (smoothly) stably isomorphic to a K-oriented normal map because we can replace if needed $E$ by $E \oplus E$, which is canonically equivariantly $\mathrm{K}$-oriented using the $\mathcal{G}$-equivariant complex structure, and replacing $\mathrm{N}_{X}$ by $\mathrm{N}_{X} \oplus E^{X}$.

If $\left(\mathrm{N}_{X}, \hat{g}, E\right)$ is a smooth normal map to $Z$ such that $E$ is equivariantly $\mathrm{K}$ oriented, then K-orientations on $\mathrm{N}_{X}$ are in 1-1 correspondence with K-orientations on $\mathrm{T} X$. One can prove the following.

Theorem 3.15. Let $X$ and $Y$ be smooth $\mathcal{G}$-manifolds, and assume that $X$ admits a smooth, normal $\mathcal{G}$-map to $Z$ and that $f^{*}(\mathrm{~T} Y)$ is subtrivial.

Then any smooth $\mathcal{G}$-map from $X$ to $Y$ is the trace of a smooth normal $\mathcal{G}$-map, and two smooth normal maps from $X$ to $Y$ are smoothly equivalent if and only if their traces are smoothly homotopic.

Furthermore, smooth equivalence classes of smooth K-oriented normal maps from $X$ to $Y$ are in 1-1 correspondence with pairs $(f, \tau)$ where $f$ is a smooth homotopy class of equivariant smooth map $X \rightarrow Y$ and $\tau$ is an equivariant K-orientation on $\mathrm{N}_{X} \oplus f^{*}(\mathrm{~T} X)$.

We sketch the existence part of this proof. Fix a smooth equivariant normal map $\left(\mathrm{N}_{X}, \hat{g}, E\right)$ from $X$ to $Z$, with $E$ equivariantly K-oriented. Let $Y$ be another smooth $\mathcal{G}$-manifold and $f: X \rightarrow Y$ be a smooth map. Let $g: X \rightarrow \mathrm{N}_{X} \hookrightarrow E$ the composite smooth embedding.

One obtains a a smooth embedding $X \rightarrow Y \times E=E^{Y}, x \mapsto(f(x), g(x))$. It has a (smooth) normal bundle $V$ with a smooth open embedding in $E^{Y}$. Thus, one gets a smooth normal map of the form $(V, E, \hat{f})$ with trace $f$ and stable normal bundle $[V]-\left[E^{X}\right] \in \mathrm{VK}_{\mathcal{G}}(X)$.

One easily checks from the given information that (1) $V \cong \mathrm{N}_{X} \oplus f^{*}(\mathrm{~T} Y)$, and (2) $V \oplus \mathrm{T} X \cong f^{*}(\mathrm{~T} Y) \oplus E^{X}$. In particular the subtriviality of $\mathrm{N}_{X}$ and $f^{*}(\mathrm{~T} Y)$ imply that of $V$; of course subtriviality of $\mathrm{T} X$ already follows from the existence of the smooth normal map from $X$ to $Z$.

The stable normal bundle is $:=[V]-\left[E^{X}\right] \in \mathrm{VK}_{\mathcal{G}}(X)$ and from (2) above this is $=f^{*}([\mathrm{TY}])-[\mathrm{T} X]$ which does not depend on the choice of normal structure. In fact, the point of the theorem is that the normal map itself only depends on the smooth structures on $X$ and $Y$, and on the smooth map $f$. 
By (1) and (2), equivariant K-orientations on $V$ are in 1-1 correspondence with equivariant K-orientations on $\mathrm{N}_{X} \oplus f^{*}(\mathrm{TY})$, and these are in turn in 1-1 correspondence with K-orientations on $\mathrm{T} X \oplus f^{*}(\mathrm{~T} Y) \oplus E^{X}$.

3.5. Equivariant K-theory. A correspondence consists, roughly speaking, of a normal map, an ordinary map, and a K-theory class. One of the significant ways in which our framework of equivariant correspondences differs from that of Baum, Connes, and Skandalis, is in the type of K-theory class we allow. The kind of $\mathrm{K}$-theory which is appropriate for our purposes is a relative version of equivariant representible K-theory. One could try to use bundles instead as K-theory data, but in addition to the usual difficulties with a lack of equivariant vector bundles, this restricts the way one handles non-proper maps.

In this section, we present an exceedingly brief overview of equivariant K-theory, roughly sufficient for the theory of equivariant correspondences. For more details see 12 .

The following discussion applies to general locally compact groupoids $\mathcal{G}$ with Haar system, and not just proper ones, but we usually consider only proper $\mathcal{G}$ spaces anyway and so we retain the hypothesis that $\mathcal{G}$ is proper for unity with the rest of the presentation.

Let $X$ be a $\mathcal{G}$-space. Recall that a $\mathcal{G} \ltimes X$-space consists of a $\mathcal{G}$-space $Y$ together with a $\mathcal{G}$-equivariant map $\varrho_{Y}: Y \rightarrow X$ serving as the anchor map for the $\mathcal{G} \ltimes X$ action.

Definition 3.16. Let $Y$ be a $\mathcal{G} \ltimes X$-space. The $\mathcal{G}$-equivariant representible $\mathrm{K}$-theory of $Y$ with $X$-compact supports is the group

$$
\operatorname{RK}_{\mathcal{G}, X}^{-*}(Y):=\mathrm{KK}_{*}^{\mathcal{G} \ltimes X}\left(\mathcal{C}_{0}(X), \mathcal{C}_{0}(Y)\right) .
$$

The $\mathcal{G}$-equivariant representible $\mathrm{K}$-theory of $Y$ is

$$
\operatorname{RK}_{\mathcal{G}}^{*}(Y):=\operatorname{RK}_{\mathcal{G}, Y}^{*}(Y) .
$$

Cycles for $\mathrm{KK}^{\mathcal{G} \ltimes X}\left(\mathcal{C}_{0}(X), \mathcal{C}_{0}(Y)\right)$ consist of pairs $(\mathcal{H}, F)$ where $\mathcal{E}$ is a countably generated $\mathbb{Z} / 2$-graded $\mathcal{G} \ltimes X$-equivariant right Hilbert $\mathcal{C}_{0}(X)$-module equipped with a $\mathcal{G} \ltimes X$-equivariant ${ }^{*}$-homomorphism from $\mathcal{C}_{0}(X)$ to the $\mathrm{C}^{*}$-algebra of bounded, adjointable operators on $\mathcal{E}$, and $F$ is a bounded, odd, self-adjoint, essentially $\mathcal{G}$ equivariant adjointable operator on $\mathcal{H}$ satisfying the usual conditions of equivariant Kasparov theory that $f\left(F^{2}-1\right)$ is a compact operator, for all $f \in \mathcal{C}_{0}(X)$. We note immediately that properness of $\mathcal{G}$ implies that $F$ may be averaged to be actually $\mathcal{G}$-equivariant, so we assume this in the following.

The Hilbert $\mathcal{C}_{0}(Y)$-module $\mathcal{E}$ is the space of continuous sections of a continuous field of $\mathbb{Z} / 2$-graded Hilbert spaces $\left\{\mathcal{H}_{y} \mid y \in Y\right\}$ over $Y$. Since $F$ must be $\mathcal{C}_{0}(Y)$ linear, it consists of a continuous family $\left\{F_{y} \mid y \in Y\right\}$ of odd operators on these graded Hilbert spaces such that $F_{y}^{2}-1$ is a compact operator on $\mathcal{H}_{y}$ for all $y \in Y$.

By $\mathcal{G} \ltimes X$-equivariance, the representation of $\mathcal{C}_{0}(X)$ on $\mathcal{E}$ must factor through the ${ }^{*}$-homomorphism $\mathcal{C}_{0}(X) \rightarrow \mathcal{C}_{0}(Y)$ Gelfand dual to the anchor map $\varrho_{Y}: Y \rightarrow X$. Therefore $F$ is $\mathcal{C}_{0}(X)$-linear as well; more, the induced representation of $\mathcal{C}_{0}(X)$ on each Hilbert space $\mathcal{H}_{y}$ sends a continuous function $f \in \mathcal{C}_{0}(X)$ to the operator of multiplication by the complex number $f\left(\varrho_{Y}(y)\right)$.

In particular, the only role of the representation of $\mathcal{C}_{0}(X)$ is to relax the support condition on the compact-operator valued-function $F^{2}-1$ from requiring it to vanish at $\infty$ of $Y$ to only requiring it to vanish at infinity along the fibres of $\varrho_{Y}: Y \rightarrow X$.

Example 3.17. If $\varrho_{Y}: Y \rightarrow X$ is a proper map then $\operatorname{RK}_{\mathcal{G}, X}^{*}(Y)=\operatorname{RK}_{\mathcal{G}}^{*}(X):=$ $\mathrm{RK}_{\mathcal{G}, X}^{*}(X)$; these two groups have exactly the same cycles. 
Any $\mathcal{G}$-equivariant complex vector bundle $V$ on $Y$ yields a cycle for $\operatorname{RK}_{\mathcal{G}}^{0}(Y)$ by choosing a $\mathcal{G}$-invariant Hermitian metric on $V$ and forming the corresponding $\mathcal{G} \ltimes Y$-equivariant $\mathbb{Z} / 2$-graded right Hilbert $\mathcal{C}_{0}(Y)$ module of sections. We may set the operator equal to zero (and the grading to be the trivial grading).

Example 3.18. Let $X$ be a $\mathcal{G}$-space and let $V$ be a $\mathcal{G}$-equivariantly K-oriented vector bundle over $X$ of (real) dimension $n$. The vector bundle projection $\pi_{V}: V \rightarrow X$ gives $V$ the structure of a space over $X$, so that $V$ becomes a $\mathcal{G} \ltimes X$-space. Then the Thom isomorphism provides an invertible Thom class

$$
t_{V} \in \mathrm{RK}_{\mathcal{G}, X}^{-\operatorname{dim} V}(V):=\operatorname{KK}_{\operatorname{dim} V}^{\mathcal{G} \times X}\left(\mathcal{C}_{0}(X), \mathcal{C}_{0}(V)\right)
$$

In the case $\mathcal{G}=\operatorname{Spin}^{c}\left(\mathbb{R}^{n}\right)$ and $X=\star$ and $V:=\mathbb{R}^{n}$ with the representation $\operatorname{Spin}^{c}\left(\mathbb{R}^{n}\right) \rightarrow \operatorname{Spin}\left(\mathbb{R}^{n}\right) \rightarrow \mathrm{O}(n, \mathbb{R})$ the class $t_{\mathbb{R}^{n}}$ is the 'Bott' class figuring in equivariant Bott Periodicity.

Certain further normalizations can be made in order to describe the groups $\operatorname{RK}_{\mathcal{G}, X}^{*}(Y)$. A standard one is to replace the $\mathbb{Z} / 2$-grading on $\mathcal{E}$ by the standard even grading, so that $\mathcal{E}$ consists of the sum of two copies of the same Hilbert module. This means that $F$ can be taken to be of the form $\left(\begin{array}{cc}0 & F_{1}^{*} \\ F_{1} & 0\end{array}\right)$ and the conditions involving $F$ are replaced by ones involving $F_{1}$ and $F_{1}^{*}$; we may as well replace $F$ by $F_{1}$. With this convention, the Fredholm conditions are that $f\left(F F^{*}-1\right)$ and $f\left(F^{*} F-1\right.$ are compact for all $f \in \mathcal{C}_{0}(X)$. In other words, $y \mapsto F_{y}$ takes essentially unitary values in $\mathbb{B}\left(\mathcal{H}_{y}\right)$ for all $y \in Y$ and the compact-operator-valued functions $F F^{*}-1$ and $F^{*} F-1$ vanish at infinity along the fibres of $\varrho: Y \rightarrow X$.

The equivariant stabilization theorem for Hilbert modules implies that we may take $\mathcal{H}$ to have the special form $L^{2}(\mathcal{G})^{\infty} \otimes_{\mathcal{C}_{0}(Z)} \mathcal{C}_{0}(Y)$, where $L^{2}(\mathcal{G})$ is the $\mathcal{G}$ equivariant right Hilbert $\mathcal{C}_{0}(Z)$ - Hilbert module defined using the Haar system of $\mathcal{G}$, and the superscript indicates the sum of countably many copies of $L^{2}(\mathcal{G})$. The corresponding field of Hilbert space has value $L^{2}\left(\mathcal{G}^{y}\right)^{\infty}$ at $y \in Y$ where $\mathcal{G}^{y}$ denotes all $\gamma \in \mathcal{G}$ ending in $y$, on which we have a given measure specified by the Haar system of $\mathcal{G}$.

This leads to a description of $\operatorname{RK}_{\mathcal{G}, Y}^{0}(X)$ as the group of homotopy-classes of $\mathcal{G}$-equivariant continuous maps from $Y$ to the space $\mathcal{F}_{\mathcal{G}}$ of Fredholm operators on the Hilbert spaces $L^{2}\left(\mathcal{G}^{y}\right)^{\infty}$, but topologizing the space $\mathcal{F}_{\mathcal{G}}$ is somewhat delicate. Similarly, the relative groups $\operatorname{RK}_{\mathcal{G}, X}^{*}(Y)$ are maps to Fredholm operators with compact vertical support with respect to the map $Y \rightarrow X$, where the support of a map to Fredholm operators is by definition the complement of the set where the map takes invertible values. We refer the reader to [12.

3.6. Correspondences. We are now in a position to define what correspondences are. Let $\mathcal{G}$ continue to denote a proper groupoid.

Definition 3.19. Let $X$ and $Y$ be $\mathcal{G}$-spaces. A $\mathcal{G}$-equivariant correspondence from $X$ to $Y$ is a quadruple $(M, b, f, \xi)$ where $M$ is a $\mathcal{G}$-space, $f: M \rightarrow Y$ is a $\mathcal{G}$ equivariantly K-oriented normal map, $b: M \rightarrow X$ is an equivariant map, and $\xi \in$ $\mathrm{RK}_{\mathcal{G}, X}^{*}(M)$ is a $\mathcal{G}$-equivariant $\mathrm{K}$-theory class with $X$-compact support, where the $\mathcal{G} \ltimes X$-structure on $M$ with respect to the $\mathcal{G}$-equivariant map $b: M \rightarrow X$. $\xi$.

The degree of the correspondence $(M, b, f, \xi)$ is the sum of the degrees of $f$ and

Remark 3.20. Thus a significant difference from the set-up of Connes and Skandalis in 8 is that the map $b: M \rightarrow X$ is not required to be proper; we have replaced this by a support condition on $\xi$. 
Several equivalence relations on correspondences are imposed to make them the morphisms in a category. The first is to consider two correspondences $\left(M, b_{0}, f_{0}, \xi\right)$ and $\left(M, b_{1}, f_{1}, \xi\right)$ to be equivalent if their normal maps are equivalent. The second is to consider bordant correspondences equivalent (we will not discuss this at all in this survey.) The third is most interesting, and is called Thom modification. The Thom modification of a correspondence $(M, b, f, \xi)$ using a subtrivial K-oriented vector bundle $V$ over $M$ is the correspondence

$$
\left(V, b \circ \pi_{V}, f \circ \pi_{V}, \tau_{V}(\xi)\right),
$$

where $\tau_{V}: \operatorname{RK}_{\mathcal{G}, X}^{*}(M) \stackrel{\cong}{\rightarrow} \mathrm{RK}_{\mathcal{G}, X}^{*-\operatorname{dim}}(V)(V)$ is the Thom isomorphism, i.e. $\tau_{V}(\xi):=$ $\pi_{V}^{*}(\xi) \cdot \xi_{V}$, where $\xi_{V} \in \mathrm{RK}_{\mathcal{G}, M}^{-\operatorname{dim}(V)}(V)$ is the Thom class. We declare a correspondence and its Thom modification to be Thom equivalent. Note that applying Thom modification to a correspondence does not change increases its degree.

The equivalence relation on correspondences is that generated by equivalence of normal maps, bordism and Thom equivalence.

Definition 3.21. Let $\mathcal{G}$ be a proper groupoid and $X$ and $Y$ be $\mathcal{G}$-spaces. We let $\widehat{\mathrm{KK}}_{*}^{\mathcal{G}}(X, Y)$ denote the set of equivalence classes of $\mathcal{G}$-equivariant correspondences from $X$ to $Y$.

A correspondence $(M, b, f, \xi)$ from $X$ to $Y$ is smooth if $X, Y$ and $M$ are smooth manifolds, $f$ is a smooth normal map (see \$3.2), and $b$ is a smooth map. There is a rather obvious notion of smooth equivalence of smooth correspondences. This gives rise to a parallel theory using only smooth equivalence classes of smooth correspondences; we do not use notation for this, but will return to the point later.

3.7. $\widehat{\mathbf{k k}}^{\mathcal{G}}$ as a category. Finally, classes of correspondences form a category. The composition of correspondences is called the intersection product. We do not describe the general intersection product here, since the correspondences we will discuss here will be all be composable by transversality (c.f. [8]).

Recall that two smooth $\mathcal{G}$-maps $f_{1}: M_{1} \rightarrow Y$ and $b_{2}: M_{2} \rightarrow Y$ are transverse if for every $\left(p_{1}, p_{2}\right) \in M_{1} \times M_{2}$ such that $f_{1}\left(p_{1}\right)=b_{2}\left(p_{2}\right)$, then $D_{p_{1}} f_{1}\left(\mathrm{~T}_{p_{1}} M_{1}\right)+$ $D_{p_{2}} b_{2}\left(\mathrm{~T} M_{2}\right)=\mathrm{T}_{f_{1}\left(p_{1}\right)}(X)$. Transversality ensures that the space

$$
M_{1} \times_{X} M_{2}:=\left\{\left(p_{1}, p b_{2}\right) \in M_{1} \times M_{2} \mid f_{1}\left(p_{1}\right)=b_{2}\left(p_{2}\right)\right\}
$$

has the structure of a smooth $\mathcal{G}$-manifold.

Theorem 3.22. Let $\Phi_{1}=\left(M_{1}, b_{1}, f_{1}, \xi_{1}\right)$ and $\Phi_{2}=\left(M_{2}, b_{2}, f_{2}, \xi_{2}\right)$ be smooth correspondences from $X$ to $Y$ and from $Y$ to $U$, respectively. Assume that both $M_{1}$ and $M_{2}$ admit smooth normal maps to $Z$ (see \$3.3), so that we lose nothing if we view $f_{1}$ and $f_{2}$ as $\mathrm{K}$-oriented smooth maps (see Theorem 3.15).

Assume also that $f_{1}$ and $b_{2}$ are transverse, so that $M_{1} \times_{Y} M_{2}$ is a smooth $\mathcal{G}$-manifold; it has a smooth normal map to $Z$ as well, and the intersection product of $\Phi_{1}$ and $\Phi_{2}$ is the class of the correspondence

$$
\left(M_{1} \times_{Y} M_{2}, b_{1} \circ \pi_{1}, f_{2} \circ \pi_{2}, \pi_{1}^{*}\left(\xi_{1}\right) \otimes \pi_{2}^{*}\left(\xi_{2}\right)\right),
$$

where $\pi_{j}: M_{1} \times_{Y} M_{2} \rightarrow M_{j}$ for $j=1,2$ are the canonical projections.

In the non-equivariant situation, any two smooth maps can be perturbed to be transverse, and in [8] this is shown to give rise to a bordism of correspondences. As a result, one can compose bordism classes of correspondence by transversality. It is important that this fails in the equivariant situation because pairs of smooth maps cannot in general be perturbed equivariantly to be transverse; this happsn in even some of the simplest situations. Consider the embedding $\star \rightarrow \mathbb{C}$ of the origin in $\mathbb{C}$. It is equivariant with respect to the $\mathbb{Z} / 2$-action by multiplication by -1 on $\mathbb{C}$. It is 
also equivariantly K-oriented, since the $\mathbb{Z} / 2$-action preserves the complex structure on $\mathbb{C}$. It is clearly normal, and it's degree is 2 .

As 0 is the only fixed-point of the $\mathbb{Z} / 2$-action, this map cannot be perturbed to be transverse to itself, because it is the unique equivariant map $\star \rightarrow \mathbb{C}$. This means that we cannot compose, for example, the correspondence $x:=[\star \leftarrow \star \rightarrow \mathbb{C}]$ and the degree 0 correspondence $y:=[\mathbb{C} \leftarrow \star \rightarrow \star]$ in the order $x y$ by transversality.

It turns out that one can often perform Thom modification in a useful way, in order to make a correspondence transverse to another one. We just explain what happens in this simple situation.

The idea is to apply Thom modification to the degree 2 correspondence $x:=$ $[\star \leftarrow \star \rightarrow \mathbb{C}]$, using the equivariantly $\mathrm{K}$-oriented vector bundle $\mathbb{C}_{\chi}$ over a point, where $\mathbb{C}_{\chi}$ is $\mathbb{C}$ with the generator of $\mathbb{Z} / 2$ again acting by multiplication by -1 .

Modification then replaces the middle space $\star$ of $x$ by $\mathbb{C}_{\chi}$ and replaces the normal map $\star \rightarrow \mathbb{C}$ by the composition $\mathbb{C}_{\chi} \rightarrow \star \rightarrow \mathbb{C}$. This latter is obviously isotopic to the identity map on $\mathbb{C}$. Finally, one adds the Thom class $t_{\mathbb{C}_{\mu}} \in \mathrm{K}_{\mathbb{Z} / 2}^{-2}\left(\mathbb{C}_{\mu}\right)$.

Thus, the Thom modification of the degree 2 correspondence $[\star \leftarrow \star \rightarrow \mathbb{C}]$ is (isotopic to) to the degree -2 correspondence $x^{\prime}=\left[\star \leftarrow\left(\mathbb{C}_{\mu}, t_{\mathbb{C}_{\mu}}\right) \stackrel{\text { id }}{\rightarrow} \mathbb{C}\right]$. The identity map is transverse to any other map, since it is a submersion. Composing $x^{\prime}$ and $y$ instead of $x y$, using transversality, we get the degree -2 correspondence

$$
x^{\prime} y=\left[\star \leftarrow\left(\star,\left.\left(t_{\mathbb{C}_{\mu}}\right)\right|_{\star}\right) \rightarrow \star\right] .
$$

The restriction of the Thom class $t_{\mathbb{C}_{\mu}}$ to the origin is just the difference $[\epsilon]-[\chi] \epsilon$ $\operatorname{Rep}(\mathbb{Z} / 2)$. It is the Euler class of the K-oriented vector bundle $\mathbb{C}_{\chi}$ over the point, e.g. the restriction of the Thom class to the zero section.

Remark 3.23. This is a special case of the excess-intersection formula of Paul Baum and Jonathon Block in [4].

With the given architecture of equivariant correspondences, a similar process can be carried out when composing two arbitrary correspondences. Namely, any equivariant correspondence $X \stackrel{b}{\leftarrow}(M, \xi) \stackrel{f}{\rightarrow} Y$, whether or not it has a smooth structure, can be Thom modified using the normal data for $f$ so that the normal map $f$ becomes a special normal submersion, the composition of an open inclusion and a vector bundle projection for a trivial vector bundle. Such a correspondence can be composed with any other one using a simple fibre-product construction.

Thus, there is of course a general, rather abstract formula for the composition product, which can be shown to agree up to equivalence with the transversality product of Connes and Skandalis.

Formally, the category $\widehat{\mathrm{KK}}^{\mathcal{G}}$ is additive, with a graded-commutative symmetric monoidal structure, and the map to $\mathrm{KK}^{\mathcal{G}}$ is compatible with these extra structures. All of this is explained in detail in [16]) and instead of going through it here, we pass right away to an interesting example of equivariant correspondences. This has its origin in the representation theory of complex semisimple Lie groups.

3.8. Equivariant correspondences and the Borel-Bott-Weil theorem. The material in this section represents joint work by the author and Robert Yuncken (see [17]).

Let $G$ be the complex, semisimple Lie group $\mathbf{S L}(n, \mathbb{C})$. A minimal parabolic in $G$ is any subgroup conjugate in $G$ to the subgroup of upper triangular matrices $P$ in $\mathcal{G}$. The Borel-Bott-Weil theorem aims to study a class of representations of $G$ called holomorphic representations on the holomorphic $G$-manifold $G / P$. For our purposes (see below) we are interested in the restrictions of these representations to the maximal compact subgroup $K:=\mathbf{S U}(n, \mathbb{C}) \subset G$ associated to the holomorphic 
action of $K$ on $G / P$. These representations are defined as follows. Let $H$ be the Cartan subgroup of diagonal matrices in $G, A$ the subgroup of matrices in $H$ with strictly positive real entries, $M=K \cap M=K \cap P$ matrices in $H$ with entries complex numbers of unit modulus, and $N$ be the subgroup of upper triangular nilpotent matrices in $G$, so that $P=M A N$. Since $G=K P, G / P \cong K / M$. A unitary group character $\mu: M \rightarrow S^{1} \subset \mathbb{C}^{*}$ extends canonically to a unitary character of $H$ by letting it be trivial on $A$, and this can be induced to a $K$ equivariant holomorphic line bundle $V_{\mu}$ on $K / M \cong G / P$. The characters $\mu \in \widehat{M}$, i.e. the points of the Pontryagin dual $\widehat{M}$ of $M$, form a group naturally represented as a lattice in $\mathfrak{g}^{*}$, the ones arises from decomposing the adjoint representation are called roots and they have the form $\mu_{i j}(m):=m_{i} m_{j}$ for a diagonal matrix $m \in M$. The induction procedure just described defines an isomorphism

$$
\bigoplus_{\mu \in \widehat{M}} \mathbb{Z} \stackrel{\cong}{\rightarrow} \operatorname{RK}_{K}^{0}(G / P):=\mathrm{KK}^{K}(\mathbb{C}, \mathcal{C}(G / P)) \text {. }
$$

It is the cohomology of the Dolbeault complex on $G / P$ twisted by the holomorphic vector bundle $V_{\mu, w}$, and viewed as a finite-dimensional virtual representation of $K$ that we are interested in. In general, this depends both on the choice of $P$, the choice of Cartan subgroup $H$. We are interested in comparing the results from leaving $H$ fixed and varying $P$. Any two parabolics containing the same $H$ are conjugate by an element of the Weyl group $W:=N_{G}(H) / H$. So we are interested in the holomorphic representations obtained in the above manner by replacing $P$ by a conjugate $P_{w}:=w P w^{-1}$, for $w \in W$.

From the point of view of index theory, the object of study, then, is the Kequivariant analytic index of the $K$-equivariant Dolbeault operator on $G / P$, twisted by $\mu$.

To formalize matters, the construction just described determines a map

$$
\gamma_{\mathrm{a}}: W \times \widehat{M} \rightarrow \operatorname{Rep}(K) .
$$

where $\gamma_{\mathrm{a}}(w, \mu) \in \operatorname{Rep}(K)$ is the representation of $K$ obtained by considering the induced action on the cohomology of the Dolbeault complex on $G / P_{w}$ twisted by the induced bundle $V_{\mu, w}$ over $G / P_{w}$. The subscript on $\gamma$ means 'analytic'.

Since $K$ acts holomorphically on $G / P_{w}$, the (real) tangent bundle to the smooth manifold $\mathrm{T}\left(G / P_{w}\right)$ has a canonical $K$-equivariant $K$-orientation. Due to Mostow's embedding theorem, $G / P_{w}$ has enough $K$-equivariant vector bundles, so the map $G / P_{w} \rightarrow \star$ to a point $\star$ is a normal $K$-equivariant map.

Due to the normal bundle of the map $G / P \rightarrow *$ being equal to $-[\mathrm{T}(G / P)]$, it is also an equivariantly $\mathrm{K}$-oriented normal map. Hence we get a $K$-equivariant correspondence $(G / K$, id, $\mathbb{C}, \star)$ from $G / P$ to a point $\star$, where $\mathbb{C}$ denotes the trivial complex line bundle on $G / P$ with trivial action of $K$.

Definition 3.24. We denote by $\left[G / P_{w}\right]_{\mu} \in \widehat{\mathrm{KK}}^{K}\left(G / P_{w}, \star\right)$ the class of the $K$ equivariant correspondence $\left(G / P_{w}\right.$, id, $\left.\star, V_{\mu, w}\right)$ from $G / P_{w}$ to a point.

Composing with the map from $G / P_{w}$ to a point we also obtain a $K$-equivariant correspondence $\left(G / P, \star, \star, V_{\mu, w}\right)$ from a point to itself whose class we denote by

$$
\gamma_{\text {top }}(w, \mu) \in \widehat{\mathrm{KK}}^{K}(\star, \star) .
$$

The Atiyah-Singer index theorem says that

$$
\mathrm{KK}^{K} \circ \gamma_{\mathrm{top}}(w, \mu)=\gamma_{\mathrm{a}}(w, \mu) \in \mathrm{KK}^{K}(\mathbb{C}, \mathbb{C}) \cong \operatorname{Rep}(K) .
$$

This vital step translates the problem of computing the map $\gamma_{\mathrm{a}}: W \times \widehat{M} \rightarrow \operatorname{Rep}(K)$ as a function of the argument $w \in W$ into the topological problem of analysing the 
function

$$
\gamma_{\text {top }}: W \times \widehat{M} \rightarrow \widehat{\mathrm{KK}}^{K}(\star, \star)
$$

as a function of its argument $w$.

We next describe some equivariant correspondences giving invertible morphisms

$$
\Lambda(\omega) \in \widehat{\mathrm{KK}}^{K}\left(G / P, G / P_{w}\right)
$$

which will intertwine relate the maps $\mu \mapsto[G / P]_{\mu}:=\left[G / P_{\text {id }}\right]_{\mu}$ and $\mu \mapsto\left[G / P_{w}\right]_{\mu}$.

Let $\Delta \subset \widehat{M}$ denote the finite set of roots. In the example $G=\mathbf{S L}(n, \mathbb{C})$ these are the characters $\mu_{i j}: M \rightarrow S^{1}, \mu_{i j}(h)=h_{i} h_{j}$ for a diagonal matrix $h$ with entries $h_{1}, \ldots, h_{n}$. They span the lattice $\widehat{M}$ and so also give a spanning set of vectors in $\mathfrak{g}^{*}$. A root $\mu_{i j}$ is positive if $i<j$. Let $\Delta^{+}$be the set of positive roots.

Given $w \in W$ we consider the direct sum of the one-dimensional representations $\oplus_{\mu \in \Delta^{+} \backslash w(\Delta)}+\mathbb{C}_{\mu}$ of $M$, a subrepresentation of the adjoint representation restricted to $M$ which induces to a $K$-equivariant vector bundle on $K / M \cong G / P_{w}$. The exponential map $K$-equivariantly intertwines the complex fibrations

$$
p_{w}: G / P \cap P_{w} \rightarrow G / P_{w}, \quad \pi_{V_{\mu, w}}: V_{\mu, w} \rightarrow G / P_{w} .
$$

This proves (c.f. Example 3.12) that the natural $K$-equivariant projection $p_{w}: G / P \cap$ $P_{w} \rightarrow G / P_{w}$ is an equivariantly K-oriented normal map and points out a canonical Thom class $t_{w} \in \mathrm{RK}_{K, G / P}^{0}\left(G / P \cap P_{w}\right)$ Of course there is also an equivariant projection map $p: G / P \cap P_{w} \rightarrow G / P$.

Definition 3.25. The Borel-Bott-Weil correspondence associated to the Weyl element $w \in W$ is the $K$-equivariant correspondence $\left(G / P \cap P_{w}, p, p_{w}, \xi_{w}\right)$ from $G / P$ to $G / P_{w}$. We denote by

$$
\Lambda(w) \in \widehat{\mathrm{KK}}^{K}\left(G / P, G / P_{w}\right)
$$

its class in geometric KK-theory.

Note that if we ignore the map $p: G / P \cap P_{w} \rightarrow G / P$ part of the correspondence $\Lambda(w)$, or more formally, compose $\Lambda(w)$ on the left with the map to a point, then the result is simply the vector bundle modification of the correspondence $\left(G / P_{w}, \star\right.$, id, $\left.\mathbb{C}\right)$ where $\mathbb{C}$ is the trivial complex line bundle on $G / P_{w}$ with the trivial action of $K$. This means that

$$
u_{*}(\Lambda(w))=[1] \in \widehat{\mathrm{KK}}^{K}\left(\star, G / P_{w}\right) .
$$

where [1] $\in \widehat{\mathrm{KK}}^{K}\left(\star, G / P_{w}\right) \cong \operatorname{RK}_{K}^{0}\left(G / P_{w}\right)$ is the unit class. Under the isomorphism $\widehat{\mathrm{KK}}_{*}^{K}(\star, \star) \cong \mathrm{KK}^{K}\left(\mathbb{C}, \mathcal{C}\left(G / P_{w}\right)\right) \cong \mathrm{K}_{0}\left(K \ltimes \mathcal{C}\left(G / P_{w}\right)\right) \cong \bigoplus_{\mu \in \widehat{M}} \mathbb{Z}$, the class [1] gets mapped to the Dirac point mass at the identity $0 \in \widehat{M}$.

The formula (3.26) in combination with Theorem 3.30 below implies the relation

$$
\gamma_{\text {top }}(w, \mu)=(-1)^{\ell(w)} \gamma_{\text {top }}(\mathrm{id}, w \cdot \mu) \in \widehat{\mathrm{KK}}_{*}^{K}(\star, \star)
$$

where $\rho$ is the sum of the positive complex roots, for $w \in W w \cdot \mu:=\mu+\rho-w(\rho)$, and $\ell: W \rightarrow \mathbb{Z}_{+}$the length function on the Weyl group using the generating set of reflections in the walls of a fundamental chamber (see [7] and [33]).

By applying the natural map $\widehat{\mathrm{KK}}^{K} \rightarrow \mathrm{KK}^{K}$ we get the following close relative of the Borel-Bott-Weil theorem.

Theorem 3.28. In the above notation, the relation

$$
\gamma_{\mathrm{a}}(w, \mu)=(-1)^{\ell(w)} \gamma_{\mathrm{a}}(\mathrm{id}, w \cdot \mu) \in \operatorname{Rep}(K)
$$

holds between the given representations of $K$.

Our main result is then the following; the proof can be found in [17. 
Theorem 3.30. Let $w \in W$ and $\mu \in \widehat{M}$. Then

$$
\Lambda(w) \otimes_{G / P_{w}}\left[G / P_{w}\right]_{\mu}=(-1)^{\ell(w)}[G / P]_{w \cdot \mu} \in \widehat{\mathrm{KK}}^{K}(G / P, \star) .
$$

Remark 3.31. The above results for $\mathrm{KK}^{K}$-equivariant theory imply analogues for $\mathrm{KK}^{G}$ because of the Baum-Connes conjecture for amenable groupoids (see [32]): the action of $G$ on $G / P$ being topologically amenable implies that the natural restriction map

$$
\mathrm{KK}^{G}(\mathcal{C}(G / P) \otimes A, B) \rightarrow \mathrm{KK}^{K}(\mathcal{C}(G / P) \otimes A, B)
$$

is an isomorphism for all $G$-C $\mathrm{C}^{*}$-algebras $A, B$.

\section{DuAlity FOR SMOOTh MANIFOLDS AND THE LEFSCHETZ MAP}

We have organized this survey around the goal of computing the Lefschetz map for smooth $\mathcal{G}$-manifolds. This problem is intertwined with that of computing equivariant KK-groups topologically, and involves some differential topology. The first job is to prove that every smooth $\mathcal{G}$-manifold has a Kasparov dual built from its vertical tangent bundle. Next, we lift this duality to the topological category $\widehat{\mathrm{KK}}^{\mathcal{G}}$ in the case of smooth manifolds of finite type (see Definition 4.5 and we get a topological model of the Lefschetz map, which can be computed easily. The same geometric hypotheses that allow this argument to be carried out imply that the map $\widehat{\mathrm{KK}}^{\mathcal{G}} \rightarrow \mathrm{KK}^{\mathcal{G}}$ is an isomorphism on both the domain and range of the Lefschetz map, so that the topological model contains all the same information as the original map. This will complete our computation of Lef for smooth $\mathcal{G}$-manifolds.

4.1. Kasparov duality with the tangent bundle. Throughout this section, we let $X$ be a smooth $\mathcal{G}$-manifold. Let $D \in \mathrm{KK}^{\mathcal{G}}\left(\mathcal{C}_{0}(\mathrm{~T} X), \mathcal{C}_{0}(Z)\right.$ be the class of the Dirac operator on the smooth $\mathcal{G}$-manifold $\mathrm{T} X$, equipped with its canonical $\mathrm{K}$-orientation from the complex structure on $\mathrm{T} X$. Let $\Theta$ be the class associated to the canonically $K$-oriented smooth embedding

$$
\delta: X \rightarrow X \times \mathrm{T} X, \quad \delta(x):=(x,(x, 0)) \in \mathrm{T}_{x} X
$$

in the usual way: the normal bundle to this embedding is $\cong \mathrm{T} X \oplus \mathrm{T} X \cong \mathrm{T}_{\mathbb{C}} X$ and thus is equivariantly K-oriented because it has a complex structure. Hence the Thom isomorphism applies. Composing with the map induced by the smooth $\mathcal{G} \ltimes X$-equivariant open embedding of the normal bundle to $X \times \mathrm{T}$ yields the required class.

The following theorem is proved in [1].

Theorem 4.1. If $X$ is a smooth $\mathcal{G}$-manifold, then the triple $\left(\mathcal{C}_{0}(\mathrm{~T} X), D, \Theta\right)$ as constructed above is a $\mathcal{G}$-equivariant Kasparov dual for $X$ in the sense of Definition 1.1

A more general result is proved that one gets a symmetric Kasparov dual; recall that this results as well in the second duality isomorphism

$$
\mathrm{KK}^{\mathcal{G}}\left(\mathcal{C}_{0}(\mathrm{~T} X) \otimes A, B\right) \cong \mathrm{KK}^{\mathcal{G} \ltimes X}\left(\mathcal{C}_{0}(X) \otimes A, \mathcal{C}_{0}(\mathrm{~T} X) \otimes_{B}\right)
$$

The latter is important for index theory, and in particular for proving that the map $\widehat{\mathrm{KK}}^{\mathcal{G}} \rightarrow \mathrm{KK}^{\mathcal{G}}$ is an isomorphism under appropriate conditions.

4.2. Kasparov duality in topological KK. If the smooth $\mathcal{G}$-manifold $X$ admits a smooth normal map $\left(\mathrm{N}_{X}, \hat{g}, E\right)$ to $Z$ then the tangent bundle $\mathrm{T} X$ and the stable normal bundle $\mathrm{N}_{X}$ are smooth complementary bundles in some trivial equivariant vector bundle (see the disscussion around Theorem 3.15. $\mathrm{T} X \oplus \mathrm{N}_{X} \cong E^{X}$. Conversely if this equation holds for some $\mathrm{N}_{X}$ then one engineers a smooth normal map to $Z$ with $\mathrm{N}_{X}$ playing the same role as above. 
From this data, we obtain smooth normal $\mathcal{G}$-maps

$$
\mathrm{N}_{X} \rightarrow Z, \quad \mathrm{~T} X \rightarrow Z .
$$

For the first, we view $\mathrm{N}_{X}$ as sitting as the zero section of $\pi_{\mathrm{N}_{X}}^{*}(\mathrm{~T} X)$. Since the normal bundle to the zero-section of a vector bundle is the vector bundle, and as

$$
\pi_{\mathrm{N}_{X}}^{*}(\mathrm{~T} X) \cong \mathrm{N}_{X} \oplus \mathrm{T} X \cong E^{X},
$$

this normal map is clear; it has stable normal bundle $\left.\pi_{\mathrm{N}_{X}}^{*}([\mathrm{~T} X])-\left[E^{X}\right]\right) \in \mathrm{VK}\left(\mathrm{N}_{X}\right)$. Similarly by reversing the roles of $\mathrm{N}_{X}$ and $\mathrm{T} X$ we construct a canonical normal map $\mathrm{T} X \rightarrow Z$ with stable normal bundle $\pi_{\mathrm{T} X}^{*}\left(\left[\mathrm{~N}_{X}\right]\right)-\left[E^{X}\right]$.

We can furthermore assume that $E$ is equivariantly K-oriented. This yields canonical K-orientations on our two normal maps to $Z$, and so we get two classes in $\widehat{\mathrm{KK}}^{\mathcal{G}}\left(\mathrm{N}_{X}, Z\right)$ and $\widehat{\mathrm{KK}}^{\mathcal{G}}(\mathrm{T} X, Z)$ respectively.

For the dual classes we consider the smooth $\mathcal{G} \ltimes X$-equivariant maps

$$
X \rightarrow X \times{ }_{Z} \mathrm{~N}_{X}, \quad X \rightarrow X \times{ }_{Z} \mathrm{~T} X,
$$

each having the same formula $\delta(x):=(x,(x, 0))$. These maps are easily seen to be $\mathcal{G} \ltimes X$-equivariant smooth, K-oriented normal maps, so they yield classes e.g. $\Theta \in \mathrm{KK}^{\mathcal{G} \ltimes X}\left(X, X \times{ }_{Z} \mathrm{~N}_{X}\right)$, and similarly for the tangent bundle.

The idea is that combining the classes in $\widehat{\mathrm{KK}}^{\mathcal{G}}\left(\mathrm{N}_{X}, Z\right)$ and $\widehat{\mathrm{KK}} \mathcal{G} \ltimes X\left(X, X \times{ }_{Z} \mathrm{~N}_{X}\right)$ from (4.2) and (4.3) respectively should yield a Kasparov dual for $X$; a complementary dual is obtained by using $\mathrm{T} X$ instead.

However, there is an issue with even defining Kasparov duality because it involves the functor denoted $T_{P}$ in the discussion of duality in \$1.1, and this functor is not defined, because the forgetful map

$$
\widehat{\mathrm{KK}}^{\mathcal{G} \ltimes X} \rightarrow \widehat{\mathrm{KK}}^{\mathcal{G}}
$$

is not defined. The problem is that a $\mathcal{G} \ltimes X$-equivariant vector bundle on $X$ is the same as a $\mathcal{G}$-equivariant vector bundle on $X$, but considered as a $\mathcal{G} \ltimes X$-equivariant bundle it is automatically trivial. Hence e.g. $\widehat{\mathrm{KK}}^{\mathcal{G}} \ltimes X(X, X)$ contains arbitrary equivariant vector bundles, while the group $\widehat{\mathrm{KK}}^{\mathcal{G}}(X, X)$ involves only subtrivial ones. The forgetful map $\mathrm{KK}^{\mathcal{G} \ltimes X} \rightarrow \mathrm{KK}^{\mathcal{G}}$ is not defined for this reason.

Furthermore, having a smooth normal map to $Z$ does not help.

Example 4.4. Let $X$ be the integers with the trivial action of the circle $\mathcal{G}$. Then $X$ admits a smooth normal map to a point, since it smoothly embeds in the trivial representation of $\mathcal{G}$ on $\mathbb{R}$, with trivial normal bundle.

However, there are examples (c.f. Example 3.4) of equivariant vector bundles on $X$ which are not subtrivial.

Definition 4.5. A smooth $\mathcal{G}$-manifold $X$ has finite type if it admits a smooth normal map to $Z$, and if every $\mathcal{G}$-equivariant vector bundle on $X$ is subtrivial.

Let $X$ be a smooth $\mathcal{G}$-manifold of finite type, and $\left(\mathrm{N}_{X}, \hat{g}, E\right)$ be smooth, $\mathrm{K}$ oriented normal map to $Z$. The stable normal bundle is $\mathrm{N}_{X}$.

Theorem 4.6. Let $X$ be a smooth $\mathcal{G}$-manifold of finite type and $\mathrm{N}_{X}$ be the stable normal bundle with respect to some smooth $\mathrm{K}$-oriented normal map $X \rightarrow Z$, with its natural structure as a smooth $\mathcal{G} \ltimes X$-manifold. Let $D \in \mathrm{KK}^{\mathcal{G}}\left(\mathrm{N}_{X}, Z\right)$ and $\Theta \in \mathrm{KK}^{\mathcal{G} \ltimes X}\left(X, X \times{ }_{Z} \mathrm{~N}_{X}\right)$ the classes constructed above from the maps (4.2) and (4.3) respectively.

Then $\left(\mathrm{N}_{X}, D, \Theta\right)$ is a Kasparov dual for $X$ in $\widehat{\mathrm{KK}}^{\mathcal{G}}$ in the sense of Definition 1.1 .

Again, the stronger result is proved in [16 that one gets a symmetric Kasparov dual; this, remember this is designed to give, as well, an isomorphism of the form

$$
\widehat{\mathrm{KK}}^{\mathcal{G}}\left(\mathrm{N}_{X} \times{ }_{Z} U, V\right) \cong \widehat{\mathrm{KK}}^{\mathcal{G} \ltimes X}\left(X \times_{Z} U, \mathrm{~T} X \times_{Z} V\right) .
$$


Remark 4.7. A similar result to Theorem 4.6 holds if $\mathrm{N}_{X}$ is replaced by $\mathrm{T} X$. The change-of-dual formulas of $\$ 1.3$ yield an invertible class $\psi \in \widehat{\mathrm{KK}} \mathcal{G}\left(\mathrm{N}_{X}, \mathrm{~T} X\right)$ intertwining the two corresponding classes $\Theta$.

It is easily checked that this class is represented by the smooth, $\mathcal{G}$-equivariant K-oriented normal map $\zeta_{\mathrm{T} X} \circ \pi_{\mathrm{N}_{X}}: \mathrm{N}_{X} \rightarrow \mathrm{T} X$ obtained by composing the bundle projection of $\mathrm{N}_{X}$ and the zero-section of $\mathrm{T} X$.

The following theorem is proved in [16].

Theorem 4.8. The natural transformation $\widehat{\mathrm{KK}}_{\mathcal{G}}^{*}(X, Y) \rightarrow \mathrm{KK}_{*}^{\mathcal{G}}\left(\mathcal{C}_{0}(X), \mathcal{C}_{0}(Y)\right)$ is invertible if $X$ is a smooth $\mathcal{G}$-manifold of finite type.

We prove this using second duality to switch the problem from bivariant to monovariant K-theory, but we omit the details here.

Paul Baum, Nigel Higson and Thomas Schick in 6] prove a related result in the case of non-equivariant K-homology, while the thesis of Jeff Raven 28 contains some of the same material done equivariantly with respect to a discrete group acting smoothly and properly: this is an excellent source for learning about the subject of equivariant Dirac operators and the like.

Both these sources, however, use a different framework for correspondences from ours.

4.3. The Lefschetz map. Let $X$ be a smooth $\mathcal{G}$-manifold of finite type. Using the Kasparov duality with the stable normal bundle or tangent bundle in $\widehat{\mathrm{KK}}^{\mathcal{G}}$ as explainedin \$4.2, we define in the same way as we did in \$1.4 the Lefschetz map in $\widehat{\mathrm{KK}}^{\mathcal{G}}$; we then obtain a topological model of Lef in the sense that it fits into a commutative diagram

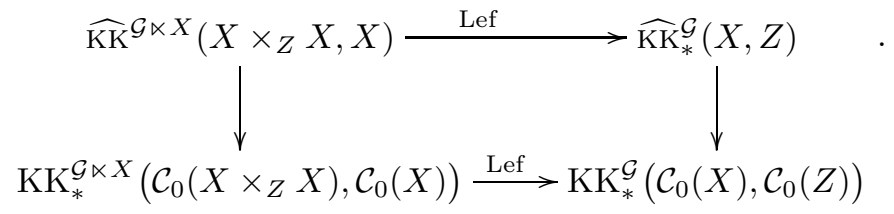

We now compute the topological model of the Lefschetz map using the tangent dual.

Start with a smooth, $\mathcal{G} \ltimes X$-equivariant correspondence

$$
\Psi:=(M, b, f, \xi), \text { or in diagram form } X \times_{Z} X \stackrel{b}{\leftarrow}(M, \xi) \stackrel{f}{\rightarrow} X
$$

from $X \times_{Z} X$ to $X$, determining a class which we also call $\Psi$ in the domain of the topological model of Lef. Recall that the $X$-structure on $X \times_{Z} X$ is on the first coordinate.

Remark 4.10. The map $f: M \rightarrow X$ embedded in the correspondence $\Psi$ is assumed a smooth normal map. This presupposes the structure on $M$ of a smooth $\mathcal{G} \ltimes X$ manifold. Note that this is a stronger condition than being a smooth $\mathcal{G}$-manifold: it entails a bundle structure on $M$ with smooth fibres.

Following the definition of the Lefschetz map in \$1.4 we next apply the functor $T_{\mathrm{T} X}: \widehat{\mathrm{KK}}^{\mathcal{G}} \rightarrow \mathrm{KK}^{\mathcal{G}}$ which sends a $\mathcal{G}$-space $W$ over $X$ to the space $W \times_{X} \mathrm{~T} X$, the latter being the same as the pullback to $W$ of the vector bundle TX using the map $W \rightarrow X$; recall that this functor is well-defined provided that every $\mathcal{G}$-equivariant vector bundle over $X$ is subtrivial.

The functor $T_{\mathrm{T} X}$ maps a $\mathcal{G} \ltimes X$-equivariant map from $W$ to $V$ to the $\mathcal{G}$-equivariant map $W \times_{X} \mathrm{~T} X \rightarrow V \times_{X} \mathrm{~T} X$ given by the obvious formula. Since $X \times_{Z} X \times_{X} \mathrm{~T} X \cong$ 
$X \times_{Z} \mathrm{~T} X$ via the map which forgets the first coordinate, applying the map $T_{\mathrm{T} X}$ to $\Psi$ yields the $\mathcal{G}$-equivariant correspondence

$$
X \times_{Z} \mathrm{~T} X \stackrel{\bar{b}}{\leftarrow}\left(M \times_{X} \mathrm{~T} X, \xi\right) \stackrel{\bar{f}}{\rightarrow} \mathrm{T} X,
$$

where $\bar{b}(m, x, \xi):=\left(b^{\prime}(m), x, \xi\right)$, where $b^{\prime}:=\operatorname{pr}_{2} \circ b$ is the second coordinate value of $b: M \rightarrow X \times{ }_{Z} X$ (since the first coordinate value is just the anchor map $\varrho_{M}^{\mathcal{G} \ltimes X}: M \rightarrow$ $X$ the map $b$ is determined by $b^{\prime}$.)

We then compose with the class of the smooth $\mathrm{K}$-oriented normal map $\mathrm{T} X \rightarrow Z$, using transversality (see Theorem 3.22) in the category of smooth, $\mathcal{G}$-equivariant correspondences: in the notation of the theorem $M_{1}:=M \times_{X} \mathrm{TX}$ and $M_{2}=$ $\mathrm{T} X$, and $f_{1}=f \times_{X}$ id: $M \times_{X} \mathrm{~T} X \rightarrow X \times_{X} \mathrm{~T} X=\mathrm{T} X$, and $b_{2}:=\mathrm{id}: \mathrm{T} X \rightarrow$ $\mathrm{T} X$. We have already argued that the projection $\mathrm{T} X \rightarrow Z$ is smooth normal, and the map $f_{1}$ is smooth and normal by assumption; furthermore, $f_{1}$ and $b_{2}$ are obviously transverse since $b_{2}$ is a submersion. We obtain the class of the $\mathcal{G}$ equivariant correspondence

$$
X \times_{Z} \mathrm{~T} X \stackrel{\bar{b}}{\leftarrow}\left(M \times_{X} \mathrm{~T} X, \xi\right) \rightarrow Z,
$$

and the only remaining step is to compose with the dual class, which is the most interesting one from our point of view.

Recall that a bar denotes forgetting the $X$-structure on a $\mathcal{G} \ltimes X$-equivariant morphism. The class $\bar{\Theta}_{\text {top }}$ that we want to compose with is that of the $\mathcal{G}$-equivariant correspondence

$$
X \stackrel{\text { id }}{\longleftarrow} X \stackrel{\delta}{\rightarrow} X \times_{Z} \mathrm{~T} X
$$

where $\delta(x):=(x,(x, 0))$. Again by Theorem 3.22 we can carry out this computation in terms of various fibre-products provided that the map $\delta$ is transverse to the map $\bar{b}$. This can be checked to be the case if, for all $m \in M$ for which $\varrho_{G}^{\mathcal{G} \ltimes X}(m)=b^{\prime}(m)$, the linear map

$$
\mathrm{T}_{m} M \rightarrow \mathrm{T}_{\varrho_{M}(m)} X, \quad \zeta \mapsto D_{m} b^{\prime}(\zeta)-D_{m} \varrho_{M}^{\mathcal{G} \ltimes X}(\zeta)
$$

is non-singular. This thus implies that the coincidence space

$$
\mathrm{F}_{\Psi}:=\left\{m \in M \mid \rho_{M}^{\mathcal{G} \ltimes X}(m)=b^{\prime}(m)\right\},
$$

is a smooth, equivariantly $\mathrm{K}$-oriented $\mathcal{G}$-manifold; in fact, more, by Theorem 3.22 it implies that the projection $\mathrm{F}_{\Psi} \rightarrow Z$ is a smooth, K-oriented normal map. Finally, it is easily checked that the restriction of $\xi$ to $\mathrm{F}$ has compact vertical support with respect to the map $\left(\varrho_{M}\right)_{\left.\right|_{\mathrm{F}_{\Psi}}}^{\mathcal{G} \ltimes X}: \mathrm{F}_{\Psi} \rightarrow X$, (this is already implied by Theorem 3.22), so we get a $\mathcal{G}$-equivariant correspondence from $X$ to $Z$ :

$$
\left(\mathrm{F}_{\Psi},\left(\varrho_{M}^{\mathcal{G} \ltimes X}\right)_{\left.\right|_{\mathrm{F}_{\Psi}}}, \varrho_{\mathrm{F}_{\Psi}}^{\mathcal{G}}, \xi\right),
$$

in the usual notation for anchor maps. We call this the coincidence cycle of $\Psi$.

Theorem 4.12. Let $\Psi \in \widehat{\mathrm{KK}}_{*}^{\mathcal{G} \ltimes X}\left(X \times_{X}, X\right)$; let

$$
\text { Lef : } \widehat{\mathrm{KK}}_{*}^{\mathcal{G}}\left(X \times_{Z} X, X\right) \rightarrow \widehat{\mathrm{KK}}_{*}^{\mathcal{G}}(X, Z)
$$

be the Lefschetz map in topological equivariant KK-theory. Then the topological Lefschetz invariant of the class of a correspondence $\Psi$ in general position in the sense explained above, is the class of the coincidence cycle of $\Psi$,

$$
\operatorname{Lef}([(M, b, f, \xi)])=\left[\left(\mathrm{F}_{\Psi},\left(\varrho_{M}\right)_{\left.\right|_{\mathrm{F}_{\Psi}}}^{\mathcal{G} \ltimes X}, \varrho_{\mathrm{F}_{\Psi}}^{\mathcal{G}}, \xi\right)\right] \in \widehat{\mathrm{KK}}_{*}^{\mathcal{G}}(X, Z) .
$$

Similar statements follow in analytic KK. 
Namely, the Lefschetz invariant of the $\mathrm{KK}^{\mathcal{G} \ltimes X}\left(X \times{ }_{Z} X, X\right)$-morphism $\mathrm{KK}^{\mathcal{G} \ltimes X}(\Psi)$ determined by $\Psi$ is the class $\mathrm{KK}^{\mathcal{G}}(\operatorname{Lef}(\Psi))$. Furthermore, this class is the pushforward under the map $\left(\varrho_{M}\right)_{\left.\right|_{F_{\Psi}}}^{\mathcal{G} \ltimes X}: \mathrm{F}_{\Psi} \rightarrow X$ of the class of the Dirac operator on the K-oriented coincidence manifold $\mathrm{F}_{\Psi}$, twisted by $\xi$. We should mention that this requires an appeal to the Index Theorem (see \$4.5).

We leave it to the reader to compute the Lefschetz invariant of $\Psi$ in the situation where the transversality condition (4.11) fails; in this case it becomes necessary to modify $\bar{\Theta}_{\text {top }}$ to make its normal component submersive and so automatically transverse to any other map. Aside from this variant, which we leave to the reader, our computation of the Lefschetz map for smooth $\mathcal{G}$-manifolds of finite type is now complete, in view of (4.9) and Theorem 4.8 (and the general result that the smooth and non-smooth theories of correspondences agree for $X$ of finite type.)

4.4. Lefschetz invariants of self-morphisms of $X$. We now consider the composition

$$
\widehat{\mathrm{KK}}^{\mathcal{G}}(X, X) \rightarrow \widehat{\mathrm{KK}}^{\mathcal{G} \ltimes X}\left(X \times_{Z} X, X\right) \stackrel{\text { Lef }}{\longrightarrow} \mathrm{KK}^{\mathcal{G}}(X, Z),
$$

where the first map is the composition of the canonical inflation map

$$
p_{X}^{*}: \widehat{\mathrm{KK}}^{\mathcal{G}}(X, X) \rightarrow \widehat{\mathrm{KK}}^{\mathcal{G} \ltimes X}\left(X \times_{Z} X, X \times_{Z} X\right)
$$

with the map

$$
\widehat{\mathrm{KK}}^{\mathcal{G} \ltimes X}\left(X \times_{Z} X, X \times_{Z} X\right) \rightarrow \widehat{\mathrm{KK}}^{\mathcal{G} \ltimes X}\left(X \times_{Z} X, X\right)
$$

of composition with the diagonal restriction class $\Delta_{X} \in \mathrm{KK}^{\mathcal{G} \ltimes X}\left(X \times_{Z} X, X\right)$. Recall that the latter is the class of the $\mathcal{G} \ltimes X$-equivariant correspondence $X \times_{Z} X \stackrel{\delta_{X}}{\longleftarrow}$ $X \stackrel{\text { id }}{\rightarrow} X$ where $\delta_{X}$ is the diagonal embedding. Let $\Psi=(M, b, f, \xi)$ be a smooth, $\mathcal{G}$-equivariant correspondence from $X$ to $X$. We note right away that this of course implies that there is a smooth normal map $M \rightarrow Z$, by composing $f: M \rightarrow X$ and $\varrho_{X}: X \rightarrow Z$. The inflation map replaces it by the $\mathcal{G} \ltimes X$-equivariant correspondence

$$
X \times_{Z} X \stackrel{\operatorname{id}_{X} \times_{Z} b}{\longleftarrow}\left(X \times_{Z} M, \xi\right) \stackrel{\operatorname{id} \times_{Z} f}{\longrightarrow} X \times_{Z} X .
$$

The $X$-structures are all on the first variable. In order to compose (on the right) with the diagonal restriction class using transversality, we first easily check that Theorem 3.22 ) applies, and deduce that we require in addition that the smooth $\operatorname{maps}_{X} \operatorname{id}_{Z} f: X \times_{X} M \rightarrow X \times_{X} X$ and $\Delta_{X}: X \rightarrow X \times_{Z} X$ be transverse in the sense of Theorem 3.22 , in the category of $\mathcal{G} \ltimes X$-equivariant smooth maps. They are transverse if and only the smooth $\mathcal{G}$-map $f: M \rightarrow X$ is a submersion. If this condition is met, then $f: M \rightarrow X$ gives $M$ not just the structure of a smooth $\mathcal{G}$-manifold, but the structure of a smooth $\mathcal{G} \ltimes X$-manifold, i.e. a bundle of smooth manifolds over $X$ with morphisms in $\mathcal{G}$ acting by diffeomorphisms between the fibres. Composing with $\Delta_{X}$ using transversality gives the $\mathcal{G} \ltimes X$-equivariant correspondence $X \times_{Z} X \stackrel{(b, f)}{\longleftarrow}(M, \xi) \stackrel{f}{\rightarrow} X$ where $(b, f)(m):=(b(m), f(m))$.

Computing the Lefschetz map as above, with the additional assumption of transversality in (4.11), we obtain the following.

Theorem 4.13. Let $X$ be a smooth $\mathcal{G}$-manifold of finite type, let $\Psi=(M, b, f, \xi)$ be a smooth, $\mathcal{G}$-equivariant correspondence from $X$ to $X$ such that $f$ is a submersion and such that for every $m \in M$ such that $b(m)=f(m)$, we have

$$
\zeta \mapsto D_{m} b(\zeta)-D_{m} f(\zeta)
$$

is non-singular. Then the Lefschetz invariant of $\Psi$ is the class of the smooth, $\mathcal{G}$ equivariant correspondence

$$
X \stackrel{b}{\leftarrow}\left(\mathrm{F}_{\Psi}^{\prime}, \xi\right) \rightarrow Z
$$


from $X$ to $Z$, where $\mathrm{F}_{\Psi}^{\prime}:=\{m \in M \mid b(m)=f(m)\}$ with its induced K-orientation, and by abuse of notation $\xi$ means its restriction of this $\mathrm{K}$-class to $\mathrm{F}_{\Psi}$.

Example 4.14. If $b: X \rightarrow X$ is a smooth $\mathcal{G}$-equivariant map, then $X \stackrel{b}{\leftarrow}(X, \xi) \stackrel{\text { id }}{\rightarrow} X$ is a smooth, zero-dimensional correspondence from $X$ to $X$. Since id: $X \rightarrow X$ is obviously a submersion, the Lefschetz invariant of the class of $\Psi$ can be computed directly in terms of transversality if the transversality condition (4.11) is met, which amounts to saying that for every $x \in X$ which is fixed by $b$, the linear map id $D_{x} b: \mathrm{T}_{x} X \rightarrow \mathrm{T}_{x} X$ is non-singular. This is the classical condition.

4.5. The index theorem. The duality computations and other work gone into developing the theory of equivariant correspondences and the natural map $\widehat{\mathrm{KK}}^{\mathcal{G}} \rightarrow$ $\mathrm{KK}^{\mathcal{G}}$ already essentially implies the Index Theorem for twisted Dirac operators on smooth, K-oriented $\mathcal{G}$ - manifolds, subject of course to the usual proviso that $X$ has finite type. We now explain this. We will assume that $X$ is a smooth and equivariantly K-oriented complete, Riemannian $\mathcal{G}$-manifold of finite type. Let

$$
D \in \mathrm{KK}_{*}^{\mathcal{G}}\left(\mathcal{C}_{0}(X), \mathcal{C}_{0}(Z)\right)
$$

be the class of the $\mathcal{G}$-equivariant Dirac operator on $X$, built in the usual way using the apparatus of self-adjoint unbounded operators (see [18 or the classic [29).

Recall that $X$ has exactly one smooth normal map to $Z$ in this situation, up to smooth equivalence: let it be $\left(\mathrm{N}_{X}, \hat{g}, E\right)$. We can assume that $E$ is equivariantly K-oriented. We have $\mathrm{N}_{X} \oplus \mathrm{T} X \cong E^{X}$ and hence K-orientations on $\mathrm{N}_{X}$ and $\mathrm{T} X$ are in 1-1 correspondence. Fix the one we have chosen above. The resulting smooth normal K-oriented map from $X$ to $Z$ is the topological Dirac class in $\widehat{\mathrm{KK}}_{*}^{\mathcal{G}}(X, Z)$. The index theorem (c.f. [19]) then asserts the following.

Theorem 4.15. Let $X$ be a smooth and equivariantly $\mathrm{K}$-oriented complete Riemannian $\mathcal{G}$-manifold of finite type. Let $D \in \mathrm{KK}_{*}^{\mathcal{G}}\left(\mathcal{C}_{0}(X), \mathcal{C}_{0}(Z)\right)$ be the class of the Dirac operator on $X$ associated to an equivariant $\mathrm{K}$-orientation on $\mathrm{T} X$. Then the natural map $\widehat{\mathrm{KK}}^{\mathcal{G}} \rightarrow \mathrm{KK}^{\mathcal{G}}$ maps the topological Dirac class to the analytic Dirac class.

The argument goes as follows. Assume that one has first proved that the triple $\left(\mathcal{C}_{0}(X), D, \Theta\right)$ is a Kasparov dual for $X$ when $\mathrm{T} X$ is equivariantly K-oriented, and $D$ is the class of the $\mathcal{G}$-equivariant Dirac operator on $X$, this analytic Kasparov cycle being constructed in the usual way. The class $\Theta$ is by definition the image in $\mathrm{KK}^{\mathcal{G} \ltimes X}$ of the class in $\widehat{\mathrm{KK}} \mathcal{G} \ltimes X$ of the correspondence $X \stackrel{\mathrm{id}_{X}}{\longleftarrow} X \stackrel{\delta_{X}}{\longleftrightarrow} X \times_{Z} X$. Since this set-up is completely analogous to the corresponding one proving duality for the tangent bundle, the reader will perhaps agree that all that is required is reading through the proof of the tangent bundle case and replacing where obvious with the spinor bundle.

Next, since $X$ has finite type, the topological and analytic models agree, and duality holds in the topological category (this has already been proved). Since the topological and analytic dualities agree on one of the fundamental classes, namely $\Theta$, they must agree on the other, too, by the general theory of abstract duals in \$1.1. This completes the proof of the index theorem for Dirac operators.

Remark 4.16. It is easy to extend the above proof to the case of twisted Dirac operators, but we omit the details.

4.6. Homological invariants for correspondences. The classical Lefschetz fixedpoint theorem describes the algebraic number of fixed-points of a map satisfying a transversality condition, to the graded trace of the induced map on K-theory, a global homological invariant. The latter of course only makes sense when the 
homology groups of $X$ have finite rank. Duality and the Universal Coefficient Theorem taken together implies this, so in particular it is the case if $X$ is a compact manifold. All the same remarks hold of course for K-theory as well.

Let $\mathcal{G}$ be just an arbitrary (that is, not necessarily proper) groupoid, and $X$ just a $\mathcal{G}$-space such that $\mathcal{G} \backslash X$ is compact.

We are going to consider the action of a morphism $f \in \mathrm{KK}^{\mathcal{G}}\left(\mathcal{C}_{0}(X), \mathcal{C}_{0}(X)\right)$ on equivariant $K$-theory or equivariant $\mathrm{K}$-homology. We are not interested in torsion, so we will just consider all K-theory and KK-groups for the rest of the discussion to be tensored with the rationals. They are thus vector spaces.

By equivariant $K$-homology we mean the functor $X \mapsto \mathrm{KK}_{*}^{\mathcal{G}}\left(\mathcal{C}_{0}(X), \mathcal{C}_{0}(Z)\right)$. By equivariant $K$-theory we mean the functor $X \mapsto \operatorname{RK}_{\mathcal{G}}^{*}(X) \cong \mathrm{K}_{*}\left(\mathcal{G} \ltimes \mathcal{C}_{0}(X)\right)$.

We are going to want to pair equivariant $\mathrm{K}$-theory classes with equivariant $\mathrm{K}$ homology classes to get integers. Since equivariant K-homology is always a module over equivariant K-theory, and we want our index map to compatible with it in the sense that $\langle a \cdot b, c\rangle=\langle a, b c\rangle$, it follows from the existence of a multiplicative unit $[1] \in \mathrm{RK}_{\mathcal{G}}^{0}(X)$ that this is equivalent to finding a map

$$
\text { Ind: } \mathrm{KK}^{\mathcal{G}}\left(\mathcal{C}_{0}(X), \mathcal{C}_{0}(Z)\right) \rightarrow \mathbb{Z}
$$

As we presumably want our construction to be functorial in $X$, this implies that it factors through the case $X=\mathcal{E} \mathcal{G}$, in which case the domain is the topological $\mathrm{K}$-theory $\mathrm{K}_{*}^{\text {top }}(\mathcal{G})$. There is no such preferred map $\mathrm{K}_{*}^{\text {top }}(\mathcal{G}) \rightarrow \mathbb{Z}$ unless $\mathcal{G}$ is a group (consider for example the case where $\mathcal{G}$ is just a space $Z$ ) so we assume this. For other reasons we will need to assume that $\mathcal{G}$ is discrete. If also $\Gamma \backslash X$ is compact, then composing the Baum-Connes map ([5]) with the trivial representation $\Gamma \rightarrow\{1\}$ then yields a map

$$
\mathrm{K}_{*}^{\mathrm{top}}(\Gamma) \rightarrow \mathrm{K}_{*}\left(C_{\max }^{*} \Gamma\right) \rightarrow \mathbb{Z} .
$$

Definition 4.17. Let $\Gamma$ be a discrete group, $X$ a proper $\Gamma$-space, such that $\Gamma \backslash X$ is compact. We denote by

$$
\text { Ind: } \mathrm{KK}^{\Gamma}\left(\mathcal{C}_{0}(X), \mathbb{C}\right) \rightarrow \mathbb{Z}
$$

the map which is the composition

$$
\mathrm{KK}^{\Gamma}\left(\mathcal{C}_{0}(X), \mathbb{C}\right) \rightarrow \mathrm{K}_{0}^{\text {top }}(\mathcal{G}) \stackrel{\mu}{\rightarrow} \mathrm{K}_{0}\left(C_{\text {max }}^{*} \Gamma\right) \stackrel{\epsilon_{*}}{\rightarrow} \mathbb{Z}
$$

where the first map is induced by the classifying map $X \rightarrow \mathcal{E} \Gamma$, the second is the Baum-Connes assembly map, and the last is the map induced by the trivial representation of $\Gamma$.

Example 4.18. For example, if $\Gamma$ is finite, then $\mathrm{K}_{*}^{\mathrm{top}}(\Gamma) \cong \operatorname{Rep}(\Gamma)$, and the map $\mathrm{K}_{0}^{\mathrm{top}}(\Gamma) \rightarrow \mathbb{Z}$ described above maps a difference of finite-dimensional $\Gamma$-representations to the difference of the multiplicities of the trivial representations that occur in them, while the map $\mathrm{KK}_{*}^{\Gamma}\left(\mathcal{C}_{0}(X), \mathbb{C}\right) \rightarrow \mathrm{K}_{*}^{\text {top }}(\Gamma)$ maps the class of an equivariant elliptic operator on $X$ (assumed compact) to its usual $\Gamma$-index, a difference of finite-dimensional representations of $\Gamma$.

Let us denote the result of multiplying the ring element $x \in \mathrm{RK}_{\Gamma}^{*}(X)$ by the equivariant K-homology class $a \in \mathrm{KK}_{*}^{\Gamma}\left(\mathcal{C}_{0}(X), \mathbb{C}\right)$ and then taking the index of the result as $(x, a) \mapsto\langle x, a\rangle$. Thus

$$
\langle x, a\rangle:=\operatorname{Ind}(x \cdot a) .
$$

We emphasise that this is not the $L^{2}$-index.

Example 4.19. Let $\Gamma$ be a finite group acting smoothly on a smooth compact manifold $X$. Then from [2], if $D$ is a $\Gamma$-equivariant elliptic operator on $X$, then there is 
a virtual character $\mathscr{I}(D, \sqcup): \Gamma \rightarrow \mathbb{C}$ having the form

$$
\mathscr{I}(D, \gamma)=\int_{\mathrm{T} X^{\gamma}} I(D, \gamma)
$$

where for each $\gamma \in \Gamma, I(D, \gamma)$ is a differential form on the fixed-submanifold $\mathrm{T} X^{\gamma}$. Furthermore, by design,

$$
\mathscr{I}(D \cdot \xi, \gamma)=\operatorname{ch}(\xi)_{\left.\right|_{\mathrm{T} X} \gamma} \cdot I(D, \gamma)
$$

for all $\xi \in \mathrm{K}_{\Gamma}^{*}(X)$, where $D \cdot \xi$ denotes the result of twisting $D$ by $\xi$. The Index Theorem states that

holds.

$$
\langle[1],[D]\rangle=\frac{1}{|\Gamma|} \sum_{\gamma \in \Gamma} \mathscr{I}(D, \gamma)
$$

Thus, at least in this case there is a local geometric formula for the index we have been describing.

The case where $\Gamma$ is infinite requires more discussion; we do not deal with this here, since there is no characteristic class formula already available in the literature, so we would have to develop it.

Let $L_{x}: \mathrm{RK}_{\Gamma}^{*}(X) \rightarrow \mathrm{RK}_{\Gamma}^{*}(X)$ denote the additive group homomorphism of $\operatorname{RK}_{\Gamma}^{*}(X)$ by multiplication by $x \in \operatorname{RK}_{\Gamma}^{*}(X)$. For $f \in \mathrm{KK}_{*}^{\Gamma}\left(\mathcal{C}_{0}(X), \mathcal{C}_{0}(X)\right)$, let $f_{*}$ denote the endomorphism of equivariant $K$-theory via the representation

$$
\begin{aligned}
\mathrm{KK}_{*}^{\Gamma}\left(\mathcal{C}_{0}(X), \mathcal{C}_{0}(X)\right) \stackrel{\text { descent }}{\longrightarrow} & \mathrm{KK}\left(\Gamma \ltimes \mathcal{C}_{0}(X), \Gamma \ltimes \mathcal{C}_{0}(X)\right) \\
& \rightarrow \operatorname{End}_{\mathbb{Z}}\left(\mathrm{K}_{*}\left(\Gamma \ltimes \mathcal{C}_{0}(X)\right)\right) \cong \operatorname{End}_{\mathbb{Z}}\left(\operatorname{RK}_{\Gamma}^{*}(X)\right) .
\end{aligned}
$$

It seems reasonable to call the linear transformation

$$
L(f): \operatorname{RK}_{\Gamma}^{*}(X) \rightarrow \mathbb{Z}, \quad L(f) x:=\operatorname{trace}_{s}\left(f_{*} \circ L_{x}\right)
$$

the Lefschetz operator of $f$. This generalizes the classical Lefschetz number $l(f):=$ $\operatorname{trace}_{s}\left(f_{*}\right)$ of $f$ in the sense that evaluating $L(f)$ at the unit $[1] \in \mathrm{RK}_{\Gamma}^{0}(X)$ recovers the Lefschetz number:

$$
L(f)([1])=l(f) .
$$

The Lefschetz operator contains more information; if for example if $f$ is an odd morphism then $\ell(f)=0$ but $L(f) \neq 0$ except in special cases.

The following Lefschetz theorem identities the Lefschetz operator with the index of the Lefschetz class: the latter can be computed geometrically in a variety of situations, leading to Lefschetz formulae.

Theorem 4.21. Let $\Gamma$ be a (possibly infinite) discrete group acting smoothly and properly on $X$. Let $f \in \mathrm{KK}_{*}^{\Gamma}\left(\mathcal{C}_{0}(X), \mathcal{C}_{0}(X)\right)$. The

$$
L(f) x=\langle x, \operatorname{Lef}(f)\rangle
$$

for every $x \in \operatorname{RK}_{\Gamma}^{*}(X)$.

If $\Gamma$ is a finite group, $X$ a smooth $\Gamma$-manifold, then the theory of correspondences applies, and all equivariant KK-morphisms $f \in \mathrm{KK}^{\Gamma}\left(\mathcal{C}_{0}(X), \mathcal{C}_{0}(X)\right)$ come from smooth, equivariant correspondences from $X$ to $X$ under the map $\widehat{\mathrm{KK}}^{\Gamma} \rightarrow \mathrm{KK}^{\Gamma}$.

Example 4.23. Let $X \stackrel{b}{\leftarrow}(M, \xi) \stackrel{f}{\rightarrow} X$ be such a correspondence, assume it is of dimension $d:=\operatorname{dim}(M)-\operatorname{dim}(X)+\operatorname{dim}(\xi)$ and denote its class by $\Psi$. Assume that the transversality assumptions in Theorem 4.13 are met. By this theorem, the coincidence space $\mathrm{F}_{\Psi}:=\{m \in M \mid b(m)=f(m)\}$ has the structure of a $\Gamma$ equivariant smooth, K-oriented manifold of dimension $d$. Therefore, we can twist the $\Gamma$-equivariant Dirac operator $D_{\mathrm{F}_{\Psi}}$ on $\mathrm{F}_{\Psi}$ by the restriction $\xi_{\left.\right|_{\mathrm{F}_{\Psi}}} \in \operatorname{RK}_{\Gamma}^{*}\left(\mathrm{~F}_{\Psi}\right)$. 
Let $\eta \in \mathrm{RK}_{\Gamma}^{*}(X)$. Then $b_{\left.\right|_{\mathrm{F}_{\Psi}}}^{*}(\eta) \in \mathrm{RK}_{\Gamma}^{*}\left(\mathrm{~F}_{\Psi}\right)$. For $\gamma \in \Gamma$ let $i_{\gamma}: \mathrm{TF}_{\Psi}^{\gamma} \rightarrow \mathrm{TF}_{\Psi}$ be the inclusion. Then by Theorem 4.21 and the characteristic class formulation of the Atiyah-Singer theorem, the action of the Lefschetz operator $L(f)$ on equivariant $\mathrm{K}$-theory is is given by the following local formula

$$
\operatorname{trace}_{s}\left(\Psi_{*} \circ L_{\xi}\right)=\frac{1}{|\Gamma|} \sum_{\gamma \in \Gamma} \int_{\mathrm{TF}_{\Psi}^{\gamma}} i_{\gamma}^{*}\left[\operatorname{ch}\left(\xi_{\left.\right|_{\mathrm{F}_{\Psi}}}\right) b_{\left.\right|_{\mathrm{F} \Psi}}^{*} \operatorname{ch}(\eta)\right] \mathscr{I}\left(D_{\mathrm{F}_{\Psi}}, \gamma\right)
$$

Thus the graded trace of the composition of $\Psi_{*}$ and $L_{\xi}$ acting on equivariant $K$ theory, only depends on the restriction of $\xi$ to the K-oriented coincidence manifold of $\Psi$.

We do not write out the formula for $\mathscr{I}$ explicitly, but refer the reader to [1].

Example 4.25. In the case where $M=X, f=\mathrm{id}$ and $b: X \rightarrow X$ is a smooth equivariant map in general position, the formula (4.24) can be simplified by linear algebra to the following sum of terms. Firstly, there is a contribution from each orbit of fixed-point of the map. If $x$ is such a fixed-point, the contribution from $x$ is given by the following sum

$$
\frac{1}{\left|\Gamma_{x}^{x}\right|} \sum_{\gamma \in \Gamma_{x}^{x}\left[g_{i}\right] \in \operatorname{Conj}\left(\Gamma_{x}^{x}\right)} \frac{1}{\left|Z_{i}\right|} \sum_{h \in Z_{i}} \operatorname{sign} \operatorname{det}\left[\left(\mathrm{id}-D_{x} f\right)_{\left.\right|_{\mathrm{Fix}(h)}}\right],
$$

where the middle sum is over a set of representatives $g_{1}, \ldots$ of the conjugacy classes in $\Gamma_{x}^{x}$, and where each inner sum is over the subgroup $Z_{i}$ which is the centralizer in $\Gamma_{x}^{x}$ of $g_{i}$. The stabilizer $\Gamma_{x}^{x}$ acts linearly on the vector space $\mathrm{T}_{x} X$ and so for any $h \in \Gamma_{x}^{x}$, the fixed-point set $\operatorname{Fix}(h)$ is a linear subspace left invariant by the linear map id $-D_{x} f$, so the determinant of this restriction is well-defined.

This formula (4.26) extends to the case of infinite-discrete groups and is worked out in 10 .

If $\Gamma$ is an infinite discrete group, $X$ a smooth $\Gamma$-space, then to compute Lefschetz invariants one replaces $\Gamma$ by the proper groupoid $\mathcal{G}:=\Gamma \ltimes \mathcal{E} \Gamma$ and $X$ by the $\mathcal{G}$ manifold $X \times \mathcal{E} \Gamma$. Theorem 4.13 provides a computation of the topological model of the Lefschetz map for $\mathcal{G}$ acting on $X \times \mathcal{E} \Gamma$ in topological terms, while the BaumConnes conjecture identifies the Lefschetz maps for $\Gamma$ acting on $X$ and for $\mathcal{G} \ltimes \mathcal{E} \Gamma$ acting on $X \times \mathcal{E} \Gamma$, so that the homological formula also applies. We leave it to the reader to formulate the result in detail.

However, what is missing from this is a characteristic-class type local formula for the index Ind: $\mathrm{KK}^{\Gamma}\left(\mathcal{C}_{0}(X), \mathbb{C}\right) \rightarrow \mathbb{Z}$ in the case of infinite discrete groups $\Gamma$, but this is not the place to formulate one.

\section{REFERENCES}

[1] M. F. Atiyah and I. M. Singer, The index of elliptic operators. I, Ann. of Math. (2) 87 (1968), 484-530. MR 0236950 (38 \#5243)

[2] The index of elliptic operators. III, Ann. of Math. (2) 87 (1968), 546-604. MR $0236952(38$ \#5245)

[3] Paul Baum and Jonathan Block, Equivariant bicycles on singular spaces, C. R. Acad. Sci. Paris Sér. I Math. 311 (1990), no. 2, 115-120 (English, with French summary).MR 1065441

[4] Excess intersection in equivariant bivariant $K$-theory, C. R. Acad. Sci. Paris Sér. I Math. 314 (1992), no. 5, 387-392 (English, with French summary). MR 1153721

[5] Paul Baum, Alain Connes, and Nigel Higson, Classifying space for proper actions and K-theory of group $C^{*}$-algebras, $C^{*}$-Algebras: 1943-1993 (San Antonio, TX, 1993), Contemp. Math., vol. 167, Amer. Math. Soc., Providence, RI, 1994, pp. 240-291. MR 1292018

[6] Paul Baum, Nigel Higson, and Thomas Schick, On the equivalence of geometric and analytic K-homology, Pure and Applied Mathematics Quarterly 3, no. 1, 1-24. Special issue in honor of Robert MacPherson, Part 3 of 3.

[7] Peter Abramenko and Kenneth S. Brown, Buildings, Graduate Texts in Mathematics, vol. 248, Springer, New York, 2008. Theory and applications. MR 2439729 
[8] A. Connes and G. Skandalis, The longitudinal index theorem for foliations, Publ. Res. Inst. Math. Sci. 20 (1984), no. 6, 1139-1183. MR 775126

[9] Siegfried Echterhoff, Heath Emerson, and Hyun Jeong Kim, KK-Theoretic duality for proper twisted actions (2006), eprint. arXiv: math/0610044

[10] _ , A Lefschetz fixed-point formula for certain orbifold $C^{*}$-algebras, J. Noncommut.Geom. (2008), To appear. arXiv: math/0610044.

[11] Heath Emerson and Ralf Meyer, Dualities in equivariant KK-theory (2007), eprint. arXiv: 0711.0025

[12] Equivariant representable K-theory, Journal of Topology (2008), To appear. arXiv: 0711.0025

[13] Euler characteristics and Gysin sequences for group actions on boundaries, Math. Ann. 334 (2006), no. 4, 853-904. MR 2209260

[14] _ Equivariant representable K-theory (2007), eprint. arXiv: 0710.1410

[15] _ Equivariant Lefschetz maps for simplicial complexes and smooth manifolds (2007), eprint. arXiv: 0711.0027

[16] _ Bivariant K-theory via correspondences (2008), eprint. arXiv: 0812.4949.

[17] Equivariant Lefschetz maps for simplicial complexes and smooth manifolds (2009), eprint.

[18] Nigel Higson and John Roe, Analytic K-homology, Oxford Mathematical Monographs, Oxford University Press, Oxford, 2000. Oxford Science Publications. MR 1817560 (2002c:58036)

[19] Gennadi G. Kasparov, The operator $K$-functor and extensions of $C^{*}$-algebras, Izv. Akad. Nauk SSSR Ser. Mat. 44 (1980), no. 3, 571-636, 719 (Russian); English transl., Math. USSRIzv. 16 (1981), no. 3, 513-572 (1981). MR 582160

[20] _ Equivariant KK-theory and the Novikov conjecture, Invent. Math. 91 (1988), no. 1, 147-201. MR 918241

[21] G. G. Kasparov and G. Skandalis, Groups acting on buildings, operator K-theory, and Novikov's conjecture, K-Theory 4 (1991), no. 4, 303-337.MR1115824 (92h:19009)

[22] Wolfgang Lück and Bob Oliver, The completion theorem in K-theory for proper actions of a discrete group, Topology 40 (2001), no. 3, 585-616. MR 1838997

[23] Wolfgang Lück and Jonathan Rosenberg, The equivariant Lefschetz fixed point theorem for proper cocompact $G$-manifolds, High-dimensional manifold topology, World Sci. Publ., River Edge, NJ, 2003, pp. 322-361. MR 2048727.

[24] _ Equivariant Euler characteristics and K-homology Euler classes for proper cocompact G-manifolds, Geom. Topol. 7 (2003), 569-613. MR 2026542

[25] Pierre-Yves Le Gall, Théorie de Kasparov équivariante et groupoïdes. I, K-Theory 16 (1999), no. 4, 361-390 (French, with English and French summaries). MR 1686846

[26] Wolfgang Lück and Bob Oliver, The completion theorem in K-theory for proper actions of a discrete group, Topology 40 (2001), no. 3, 585-616. MR 1838997

[27] George Daniel Mostow, Equivariant embeddings in Euclidean space, Ann. of Math. (2) 65 (1957), 432-446. MR 0087037

[28] Jeff Raven, An equivariant bivariant Chern character, PhD Thesis, the Pennsylvannia State University (2004). http://etda.libraries.psu.edu/theses/approved/WorldWideIndex/ETD723 /index.html.

[29] John Roe, Elliptic operators, topology and asymptotic methods, 2nd ed., Pitman Research Notes in Mathematics Series, vol. 395, Longman, Harlow, 1998. MR 1670907 (99m:58182)

[30] Juliane Sauer, K-Theory for proper smooth actions of totally disconnected groups, Highdimensional manifold topology, World Sci. Publ., River Edge, NJ, 2003, pp. 427-XII. 2048732

[31] Georges Skandalis, Kasparov's bivariant K-theory and applications, Exposition. Math. 9 (1991), no. 3, 193-250. MR 1121156

[32] Jean-Louis Tu, La conjecture de Baum-Connes pour les feuilletages moyennables, K-Theory 17 (1999), no. 3, 215-264 (French, with English and French summaries). MR 1703305 (2000g:19004)

[33] Jacques Tits, Buildings of spherical type and finite BN-pairs, Lecture Notes in Mathematics, Vol. 386, Springer-Verlag, Berlin, 1974. MR 0470099 (57 \#9866)

[34] Jean-Louis Tu, La conjecture de Novikov pour les feuilletages hyperboliques, K-Theory 16 (1999), no. 2, 129-184 (French, with English and French summaries). MR 1671260

[35] Julia Weber, The universal functorial equivariant Lefschetz invariant, K-Theory 36 (2006), no. 1-2, 169-207. MR 2274162

E-mail address: hemerson@math.uvic.ca

Department of Mathematics and Statistics, University of Victoria, PO BOX 3045 STN CSC, Victoria, B.C., CANada V8W 3P4 